\title{
Micro-scale Realization of Compliant Mechanisms: Manufacturing Processes and Constituent Materials-A Review
}

\author{
Minchang Wang ${ }^{1,2^{*}}$, Daohan Ge ${ }^{1 *}$, Liqiang Zhang ${ }^{1 *}$ and Just L. Herder ${ }^{2}$
}

\begin{abstract}
Compliant micromechanisms (CMMs) acquire mobility from the deflection of elastic members and have been proven to be robust by millions of silicon MEMS devices. However, the limited deflection of silicon impedes the realization of more sophisticated CMMs, which often require larger deflections. Recently, some novel manufacturing processes have emerged but are not well known by the community. In this paper, the realization of CMMs is reviewed, aiming to provide help to mechanical designers to quickly find the proper realization method for their CMM designs. To this end, the literature surveyed was classified and statistically analyzed, and representative processes were summarized individually to reflect the state of the art of CMM manufacturing. Furthermore, the features of each process were collected into tables to facilitate the reference of readers, and the guidelines for process selection were discussed. The review results indicate that, even though the silicon process remains dominant, great progress has been made in the development of polymer-related and composite-related processes, such as micromolding, SU-8 process, laser ablation, 3D printing, and the CNT frameworking. These processes result in constituent materials with a lower Young's modulus and larger maximum allowable strain than silicon, and therefore allow larger deflection. The geometrical capabilities (e.g., aspect ratio) of the realization methods should also be considered, because different types of CMMs have different requirements. We conclude that the SU-8 process, 3D printing, and carbon nanotube frameworking will play more important roles in the future owing to their excellent comprehensive capabilities.
\end{abstract}

Keywords: Compliant micromechanism, Manufacturing process, Constituent material

\section{Introduction}

Since the 1960s, a trend called 'miniaturization' has grown in popularity, creating many new fields, such as integrated circuits, nanotechnology, and microrobotics. Inspired by this trend, microelectromechanical systems (MEMS) have been established as an independent field to realize micro-systematic integration. After about half a century of development, MEMS technology has been successfully utilized in many commercial products, such as micro sensors, digital light processors, and

\footnotetext{
*Correspondence: wmc177@ujs.edu.cn; gedaohan@mail.ujs.edu.cn; zhanglq4158@ujs.edu.cn

${ }^{1}$ Institute of Intelligent Flexible Mechatronics, Jiangsu University,

Zhenjiang 212013, China

Full list of author information is available at the end of the article
}

micro speakers. These products have already permeated into everyday life and have fuelled a new industrial revolution [1]. Despite their enormous success, an increasing number of MEMS devices are under development and are expected to significantly impact disparate fields, such as nano- and bio-manipulation, microsurgery, microrobotics, micro fluidics, microenergy, precision mechanisms, etc. [2-7]. With respect to the mechanical domain, micromechanics can be realized by assembling different types of rigid-body joints or using compliant designs. Given that friction, wear, and ensuing failure are important issues on the microscale, and to date, no lubrication methods are available, compliant mechanisms [8-12] have been adopted in almost all successful MEMS 
devices to circumvent the challenge of rigid-body joints $[13,14]$.

Compliant mechanisms are mechanisms that achieve mobility by using elastic deformation rather than rigidbody joints. As such, these joints are replaced by slender beams or deep notches at equivalent locations [15]. Such flexure joints can only provide limited motion, but they have several significant advantages including the elimination of backlash, friction, and wear, as well as no need for lubrication. Moreover, their monolithic nature eliminates the need for assembly and accommodates micromachining processes. Moreover, bionic compliant design of micromemchanics is inherent in nature, because compliance plays a pivotal role in the realm of microorganisms, the level at which MEMS fit [16]. Therefore, towards compliance is a necessity when these mechanisms are downsized to the microscale [17].

To date, some successful compliant micromechanism (CMM) designs have been used in many commercial MEMS products. For instance, in compact MEMS multiaxis gyroscopes, the synchronous oscillation of multi proof masses relies on compliant transmission mechanisms [18]. Most of the mechanisms used in MEMS sensors do not require large deflections because they either work in an oscillation mode or have very limited response mechanical motion. However, large deflection remains an important trend in the development of CMMs to meet the demand for motion transfer or transformation. The representative examples of such CMMs include compliant motion amplifiers, bistable mechanisms, lamina emergent mechanisms (LEMs), double-V-beam suspensions, microgrippers, and positioners [16, 19-28]. To realize more sophisticated functions, these mechanisms are designed as more complicated structures instead of cantilevers or diaphragms $[29,30]$. In contrast to their oscillator-type counterparts, large deflections are usually required to fulfill the functions, for example, gripping and manipulation. To relieve stress concentration, distributed compliance is increasingly favored by designers over lumped compliance [16]. Moreover, the development of CMMs is also characterized by a tendency to incorporate new kinematic concepts (e.g., static balance), biotechnology, bionics, and robotics to adapt to the targeted applications [31, 32]. In summary, the aforementioned trends introduce bring stricter requirements to the manufacture of CMMs.

As previously indicated, CMMs have many advantages over conventional rigid-body mechanisms and are adapted to microfabrication processes. However, the utilization of compliance brings a substantial limitation to the designers. Given that such mechanisms gain mobility from flexible members, the degree to which their constituent material can deflect is a critical boundary condition. Therefore, CMMs have to be designed under the constraints imposed by the available constituent materials. Currently, silicon is the dominant material in the manufacture of CMMs, but it is not an ideal material to realize compliant mechanisms [33]. The yield strain of commonly used polysilicon is approximately $1 \%$; in contrast, polypropylene, a commonly used material in macroscale compliant mechanisms, can reach $2.5 \%$ before yielding [34, 35]. Moreover, failure occurs once silicon reaches its yield strain, whereas the maximum strain of polypropylene exceeds $10 \%-20 \%$ if plastic deformation is allowed [36]. Consequently, a notable burden is placed on designers as they attempt to achieve a specific motion with limited deflection. Apart from the large flexibility, some mechanisms used for biological manipulation or surgical purposes must be fabricated using biocompatible materials. Apparently, the interdisciplinary aspect of these applications has further confounded designers in the selection of constituent materials and manufacturing processes.

In addition to the silicon process, other processes, for example, polymer and composite processes, have been used in the fabrication of CMMs and are being rapidly developed. These emerging candidates are even more promising, but are not well known. Although microfabrication has already been reviewed in several reports, these papers focused on either micro structures or particular kinds of MEMS devices, or only examined the oscillatortype CMMs used in sensors [37-39]. As a result, these review papers do not capture the latest developments in CMM manufacturing; therefore, it is necessary to review the current status of the fabrication process of CMMs to assist designers to realize their ideas.

The aim of this report is to review the manufacturing processes and constituent materials that have been reported in the fabrication of CMMs. The key features of the manufacturing processes and mechanical properties of the materials have been compiled into tables, which are expected to help designers quickly identify suitable manufacturing processes and constituent materials to realize their design. In the early stages, this review will have potential impacts on designers. Inversely, it will also encourage process developers to adapt their processes to CMM trends. Furthermore, this interaction provoked will benefit the development of CMMs and increase their applications in the near future.

The remainder of this report is organized as follows. Section 2 outlines the methods used in the literature survey, classification, and statistical analysis, as well as the criteria used in the evaluation of the manufacturing processes and constituent materials. Section 3 presents the statistical results of the literature survey, summarizes some representative processes, and compiles the 
material properties and process features into an information table. Furthermore, the results are interpreted, and future directions and challenges in CMM fabrication are discussed. Section 4 present the main conclusions and examines their practical value on the side of the designers.

\section{Methods}

\subsection{Methods Used in Literature Survey and Classification}

The literature survey was divided into two parts. In the first part, a literature search was conducted for articles related to CMM manufacturing. The literature search covered compliant mechanisms with micro-scale features, that is, the dimension of some critical components (e.g., width of slender beams) is in the several-micron to hundred-micron range. However, oscillator-type MEMS sensors are excluded from the literature survey (e.g., accelerometers, gyroscopes, barometers, etc.), because such oscillator-type sensors are either designed as cantilever-like simple structures or undergo limited deflection. The second part involved a classification based on manufacturing processes and constituent materials.

The literature survey was conducted using Web of Science, covering journal papers, theses and conference proceedings. Four different sets of keywords were used: (1) compliant micromechanisms, (2) manufacturing processes, (3) constituent materials, and (4) application fields. An overview of the set of keywords is provided in Table 1. To optimize the search, all sets of keywords were combined and narrowed. In addition, the references of the articles were examined to identify relevant articles on the same subject. The results were filtered based on the titles of the article. Subsequently, the reduced results were filtered by reading the full article to confirm that the reported mechanism exhibited micro-scale features, and the fabrication process used was revealed. Otherwise, the articles were not used.

The search results were classified according to the manufacturing process. Eight groups were made: surface micromachining, bulk micromachining, laser micromachining, wire electrical discharge machining (EDM),
SU-8, micromolding, carbon nanotube (CNT) frameworking, and 3D printing. However, such a classification is not narrow enough to clarify the process features and material properties. Some manufacturing processes can be used for different materials, and vice versa. Moreover, the process features are determined by the process itself, as well as the material dealt with. Therefore, the results were further classified into each process group according to the constituent materials involved. In the remainder of this paper, the process features and material properties will be clarified with respect to such process-material combinations.

\subsection{Criteria Used in Result Evaluation}

To evaluate and compare these process-material combinations, the process features and material properties were extracted from the literature and collected into tables. The processes were evaluated with respect to the smallest feature resolution, maximum thickness of the structure layer, aspect ratio, fabrication deviation, and manufacturing cost, while the material properties include Young's modulus, Poisson's ratio, failure strength, and maximum allowable strain. Each criterion and its effect on CMM fabrication are described as follows.

Smallest feature resolution. The smallest feature resolution refers to the minimum length in a pattern that can be realized by a particular manufacturing process. To a large extent, this determines the precision of the process.

Maximum thickness of structure layer. Most CMMs have planar structures. Therefore, the thickness of the structures that can be realized is another key parameter. The thickness of the structure layer is closely related to the process. Normally, the process has a thickness limitation for the structures.

Aspect ratio. The aspect ratio is defined as the ratio between the maximum structure thickness and the smallest feature resolution. As a key feature of planar mechanisms, it determines the ratio of in-plane stiffness to out-of-plane stiffness. Aspect ratios higher than 20 are defined as high aspect ratios.

Table 1 Overview of the sets of keywords used in the literature survey

\begin{tabular}{ll}
\hline Sets & Keywords \\
\hline (1) Compliant micromechanisms & • Compliant, flexible, flexure, monolithic \\
& $\begin{array}{l}\text { - Micro, MEMS } \\
\text { - Mechanism, structure, design, gripper, manipulator, bistable, lamina emergent, motion amplifier, micro transmission }\end{array}$ \\
(2) Manufacturing processes & $\cdot$ Surface, MUMPs, SUMMiT, bulk, wire EDM, laser, molding, LIGA, UV-LIGA, DRIE, 3D printing \\
(3) Constituent materials & - Silicon, polysilicon, SU8, PDMS, aluminum, carbon nanotube \\
(4) Application fields & $\cdot$ Composite, polymer \\
\hline
\end{tabular}


Fabrication deviation. The fabrication deviation determines the accuracy of the process. A fabrication deviation of less than $5 \%$ is defined as 'low,' whereas values larger than 5\% are defined as 'high'.

Manufacturing cost. The manufacturing costs of all processes were estimated and labelled as 'high' and 'low' by relative comparison.

Young's modulus and Poisson's ratio. Young's modulus and Poisson's ratio are the most important mechanical properties in the design of compliant mechanisms. Normally, a low Young's modulus is favored, because it is proportional to the required actuation effort if the deflection remains constant.

Failure strength. The failure strength is defined as the stress at which failure occurs. The definition is subjected to great variations in implementation; for instance, the failure strength is not only determined by the material itself, but also by the manufacturing process used. To account for this, we chose to list the most widely accepted values.

Maximum allowable strain. The maximum allowable strain is one of the most important boundary conditions for CMM designers because it directly determines the largest deformation that can be achieved. Typical values of some widely used materials such as silicon, have been reported in the literature. The maximum allowable strains of the other materials were obtained by dividing the failure strength by Young's modulus.

\section{Results and Discussion}

\subsection{State of the Art in CMM Fabrication}

Approximately 170 papers on CMM manufacturing were found. Various processes have been used in the fabrication of CMMs, including surface micromachining, bulk micromachining, laser micromachining, wire EDM, micromolding, SU-8, CNT frameworking, and $3 \mathrm{D}$ printing. Figure 1 (a) shows the results of a statistical analysis of the reported papers with respect to the manufacturing process. It is evident that the silicon process is the most widely used fabrication method for CMMs. Approximately $2 / 3$ of the CMMs reported in these papers are fabricated using the silicon process, either through surface micromachining or bulk micromachining. This is closely related to the fully developed silicon MEMS technology and complete silicon processing infrastructure. However, other processes also account for a large percentage, which is indicative of their contribution to the development of CMM manufacturing. Laser ablation and wire EDM have also been used to realize some mechanisms with micro-scale features, although conventionally, they are associated with macro-scale features. In addition, micro molding and SU-8 processing have also been frequently used

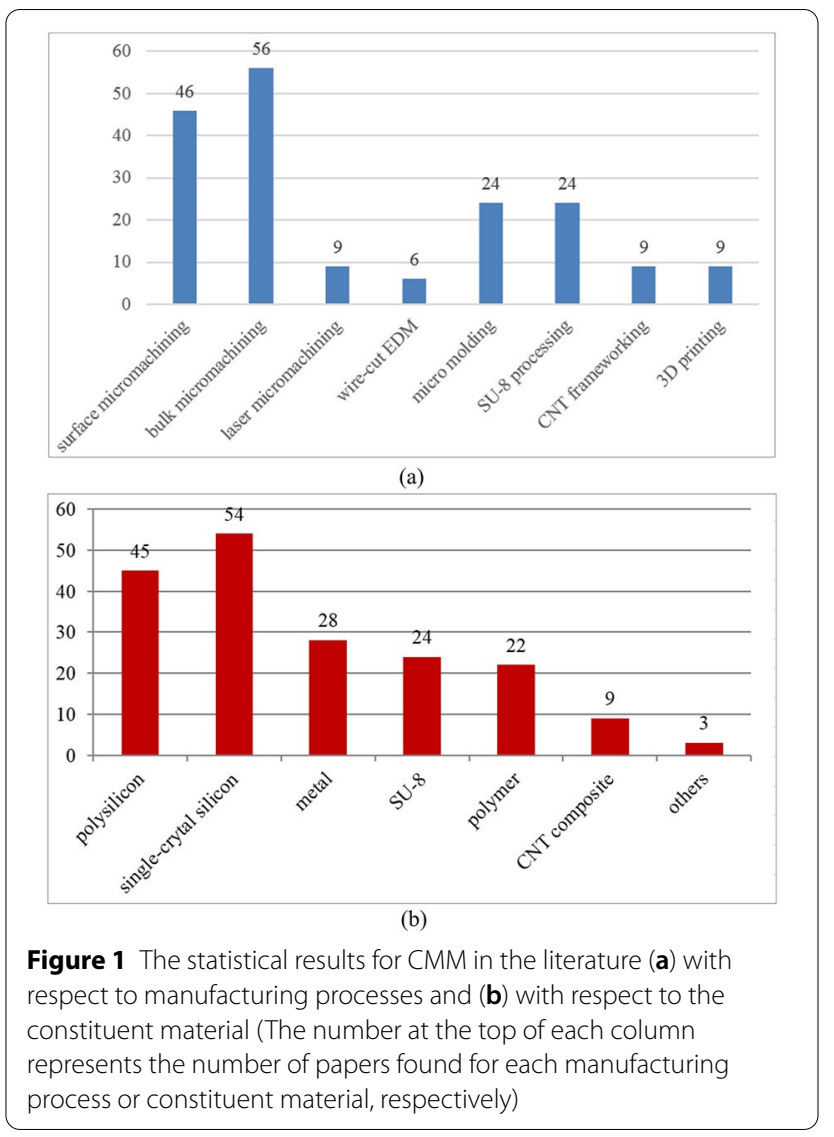

for the fabrication of CMMs. It is worth noting that in recent years, $\mathrm{CNT}$ frameworking and $3 \mathrm{D}$ printing have emerged as techniques that facilitate additional process capability with extraordinary material properties. Another assertion is that the manufacture of CMM is associated with much more diversity than conventional oscillator-type MEMS sensors.

The search results were also statistically analyzed with respect to the constituent materials used (Figure 1(b)). Unsurprisingly, polysilicon and single-crystal silicon combined account for nearly $2 / 3$ of all the papers. In addition, silicon, metal, and metal alloys have the largest fraction, which is used in laser ablation, wire EDM, and micro molding. Moreover, in CMM manufacturing, there is a strong trend towards the utilization of polymers and composites, which results in a much lower Young's modulus and larger maximum allowable strain. Some photopolymers, such as SU-8, R11, and IP-Dip, are preferred for the manufacture of CMMs because of their mechanical properties and ease of fabrication. CNT composites that result from the CNT frameworking exhibit extraordinary performance in compliant deformation due to the reinforcement of carbon nanotubes. From the perspective of constituent materials, non-silicon materials are 
being rapidly developed, even though silicon is still the most frequently used material.

\subsection{Summary of Representative Processes}

To provide a basic understanding of the differences and similarities of the various processes and the commonly used materials, eight groups are identified: surface micromachining, bulk micromachining, laser micromachining, wire EDM, micromolding, SU-8 process, CNT frameworking, and 3D printing. The essential features of each group are presented below.

\subsubsection{Surface Micromachining}

Surface micromachining is an additive process in which microstructures are formed by thin film deposition, followed by selective etching. Some commercialized surface micromachining processes, such as MUMPs and SUMMiT, have already resulted in several excellent rigid-joint micromechanisms, such as hinges, joints, and gear trains [11]. These processes are also highly favored in the realization of compliant mechanisms [40]. Unsurprisingly, polysilicon is the most widely used structural material for surface micromachining. Conventional structural polysilicon layers are very thin, typically less than $10 \mu \mathrm{m}$, because of the limitations of the film deposition technique. Indeed, the thickness increased to $15-50 \mu \mathrm{m}$ after the development of epi-poly technology, but it is mainly used in the fabrication of oscillator-type sensors [41]. Therefore, we concentrate on CMM fabrication based on two commercialized processes, MUMPs and SUMMiT.

Figure 2 shows the images of some CMMs fabricated using MUMPs and SUMMiT. The process features and

Table 2 Overview of the essential features of the SUMMiT and MUMPs processes

\begin{tabular}{lllll}
\hline $\begin{array}{l}\text { Proc. } \\
\text { feature }\end{array}$ & $\begin{array}{l}\text { Fabric. } \\
\text { resolution } \\
(\boldsymbol{\mu m})\end{array}$ & $\begin{array}{l}\text { Thickness } \\
(\boldsymbol{\mu \mathrm { m }})\end{array}$ & Aspect ratio & $\begin{array}{l}\text { Fabric. dev. } \\
(\boldsymbol{\mu \mathrm { m } )})\end{array}$ \\
\hline SUMMiT & $0.8[43]$ & $1-6.5$ & $<10$ & $0.1[46]$ \\
MUMPs & $2[20]$ & $1.5-3.5$ & $<2$ & $0.2[48]$ \\
\hline
\end{tabular}

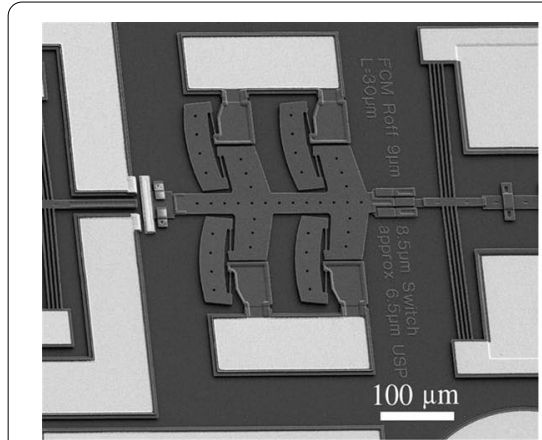

(a)

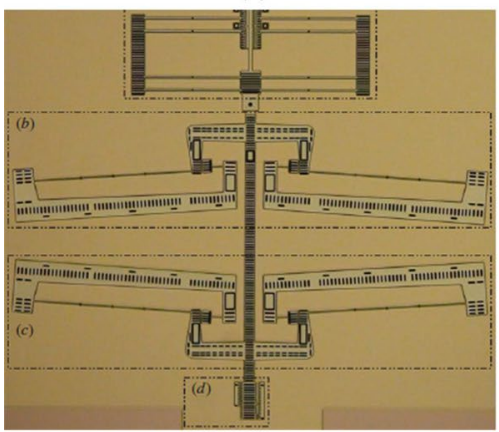

(d)

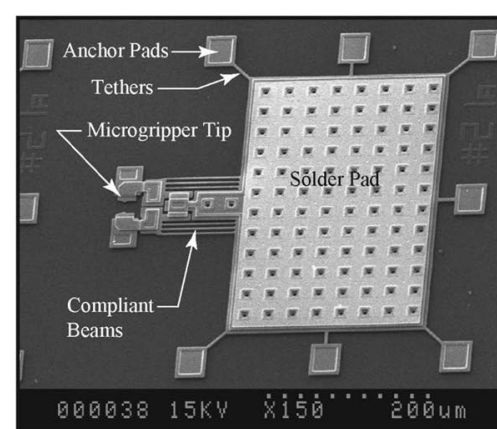

(b)

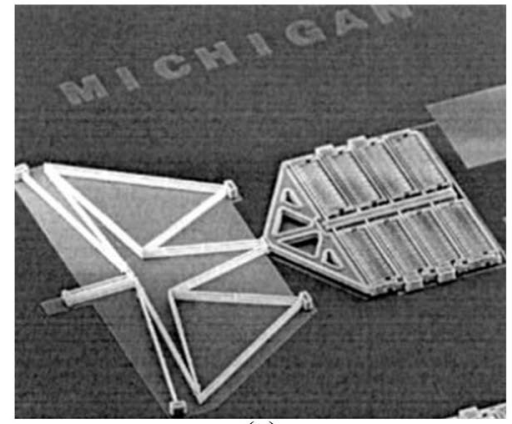

(e)

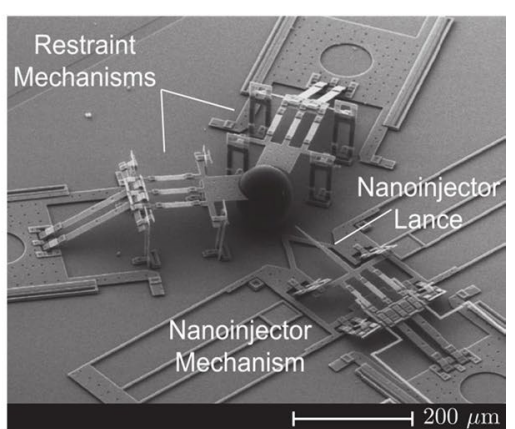

(c)

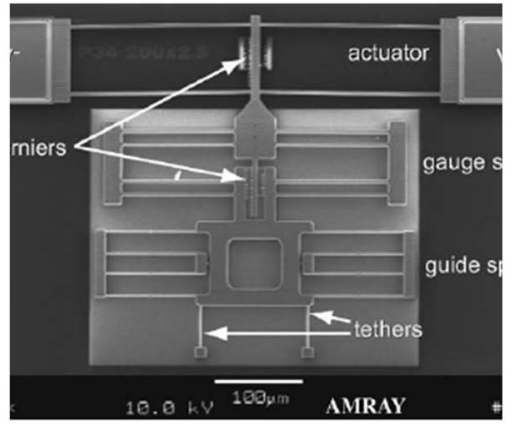

(f)

Figure 2 Some CMMs fabricated by MUMPs $(\mathbf{a}, \mathbf{b}, \mathbf{c})$ and SUMMiT ( $\mathbf{d}, \mathbf{e}, \mathbf{f})$ : (a) The self-retracting fully compliant bistable mechanism by Masters et al. [20] (OIEEE. Adapted by permission from IEEE. Permission to reuse must be obtained from the rightsholder), (b) The compliant microgripper by Dechev et al. [50] (OIEEE. Adapted by permission from IEEE. Permission to reuse must be obtained from the rightsholder), (c) The self-reconfiguring metamorphic nanoinjector by Aten et al. [42] (@AIP. Adapted by permission from AIP. Permission to reuse must be obtained from the rightsholder), (d) The stroke amplifier with electrostatic actuator combined, by Kota et al. [51] (IEEE. Adapted by permission from IEEE. Permission to reuse must be obtained from the rightsholder), (e) The thermal actuator with force gauge attached, by Wittwer et al. [19] (@Elsevier. Adapted by permission from Elsevier. Permission to reuse must be obtained from the rightsholder), (f) The fully compliant double tensural tristable micromechanism by Chen et al. [40] (@IOP. Adapted by permission of IOP. Permission to reuse must be obtained from the rightsholder) 
resultant material properties are compared in Tables 2 and 3, respectively. Both the MUMPs and SUMMiT processes can provide multilevel polysilicon capabilities. The MUMPs process has three polysilicon layers, whereas SUMMiT has five layers. The multilevel capability and extremely small feature resolution enable the realization of sophisticated CMMs, and even partially compliant mechanisms. However, these commercialized processes provide limited flexibility and impose considerable constraints on designers [42, 43]. For example, each layer has a fixed thickness that cannot be changed freely. Given that the polysilicon layers used are usually $1-3 \mu \mathrm{m}$ thick, it is difficult to fabricate highaspect-ratio structures using these such processes, even if the thickness can be increased by stacking neighboring poly layers [20]. The insufficient thickness could cause some parasitic out-of-plane motion when undergoing disturbance, but it is highly favored by some mechanisms with out-of-plane motion. For instance, Tsang et al. used MUMPs to fabricate a hingeless $90^{\circ}$ out-of-plane micromechanism called a 'Tsang mechanism,' which was fabricated in-plane and flipped upwards to the vertical position after fabrication [44].

From the viewpoint of material properties, polysilicon is not a perfect material for compliant mechanisms, especially for those requiring large deflections. Its comparatively large Young's modulus results in considerable stiffness; therefore, many researchers have incorporated thermal actuators into their design to achieve a large actuation force [45]. More importantly, the maximum allowable strain of polysilicon is too small to meet the requirements of many CMMs, and failure occurs once the maximum strain exceeds its yield strain. For MUMPs, a maximum allowable strain of $1.05 \%$ is assumed by many users, while for SUMMiT, a value of $0.6 \%$ is used [46-48], but without information provided officially. In some cases, to achieve the desired performance, CMM designers must create their designs on the verge of the material boundary conditions. For instance, in the compliant bistable mechanism designed by Jensen et al., the simulation indicates a maximum strain of $1.02 \%$, which is close to the maximum allowable strain in MUMPs. This substantially increases the risk of failure of the fabricated mechanisms [49].

\subsubsection{Bulk Micromachining}

Unlike surface micromachining, bulk micromachining defines structures by selectively etching inside a substrate. Normally, it refers to the fabrication process with silicon wafers, although a similar process has been used on polyethylene terephthalate (PET) [52]. Bulk micromachining usually requires a high-aspect-ratio etching technique, such as deep reactive ion etching (DRIE), owing to the thickness of the silicon wafer. The mechanisms thus benefit from the material properties of single-crystal silicon (SCS), which has a larger maximum allowable strain than polysilicon. The Young's modulus of SCS ranges from $150 \mathrm{GPa}$ to $170 \mathrm{GPa}$, and the widely accepted value of its fracture strength is $7 \mathrm{GPa}$, which corresponds to the maximum allowable strain of $~ 4 \%$ [53]. However, the SCS mechanisms can only achieve a maximum strain of $1 \%-2 \%$ before fracture. This considerable discrepancy is not only related to the stochastic nature of the strength of such brittle materials, but also the imperfect sidewalls resulting from DRIE [54].

One of the biggest advantages of bulk micromachining is that it facilitates a high aspect ratio. By etching through a silicon wafer, micromechanisms with a thickness of several hundred microns and aspect ratios as high as 20 can be achieved. In the most advanced DRIE facilities, an aspect ratio of 30 can be achieved. A thinner mechanism can be enabled by thinning the silicon wafer, but this is associated with enormous risks and uncertainties. Silicon on insulator (SOI) is a reliable technique that enables the formation of micromechanisms with precise and customized thickness. In particular, the SOI wafer contains three layers: a device silicon layer, a buried oxide layer, and a handle silicon layer. Normally, mechanisms are made by etching the device layer, whereas the handle layer is removed at the end or used as a support. Benefitting from the SOI technique, bulk micromachining enables the realization of CMMs with a considerable thickness range, from several microns to several hundred microns. Moreover, bulk micromachining also allows a small feature size, typically down to $1-3 \mu \mathrm{m}$. With the development of DRIE, the accuracy of the fabrication process has also steadily increased. To date, the most advanced DRIE facility can render an undercutting of less than $0.1 \mu \mathrm{m}$ and a taper angle of less than $0.1^{\circ}$.

The reported CMMs fabricated via bulk micromachining cover many different categories, for example, nanopositioners, microgrippers, micromanipulators,

Table 3 Overview of the constituent material properties of the SUMMiT and MUMPs processes

\begin{tabular}{llllr}
\hline Mater. Prop. & Young's modulus (GPa) & Poisson's ratio & Yield stress (GPa) & Yield strain (\%) \\
\hline SUMMiT & $164 \pm 3.2[46]$ & $0.23[46]$ & $1[46]$ & 0.6 \\
MUMPs & $158 \pm 10[44]$ & $0.22 \pm 0.01[44]$ & $1.65 \pm 0.28[48]$ & $\sim 1.05$ \\
\hline
\end{tabular}


bistable mechanisms, and motion amplifiers [26, 28, $55,56,58-61]$. Owing to the high maturity of the silicon process, a variety of actuation methods can be incorporated into bulk micromachined CMMs, for example, electrostatic actuation, electrothermal actuation, piezoelectric actuation, and shape memory alloy (SMA) actuation. Representative CMMs fabricated using bulk micromachining are shown in Figure 3.

\subsubsection{Laser Micromachining}

Laser machining utilizes concentrated laser energy for ablation, drilling, cutting, welding, and other modification of materials. The advantages of this process include its versatility and environmental friendliness. Almost all light-absorbing materials can be treated by laser machining. Such a process can be used in applications where feature sizes are on the microscale. The materials that have been used to fabricate CMMs include sheet metals, polymers, and ceramics. Three types of lasers are mainly used: $\mathrm{CO}_{2}$ lasers, $\mathrm{Nd}$ :YAG lasers, and excimer lasers. Normally,

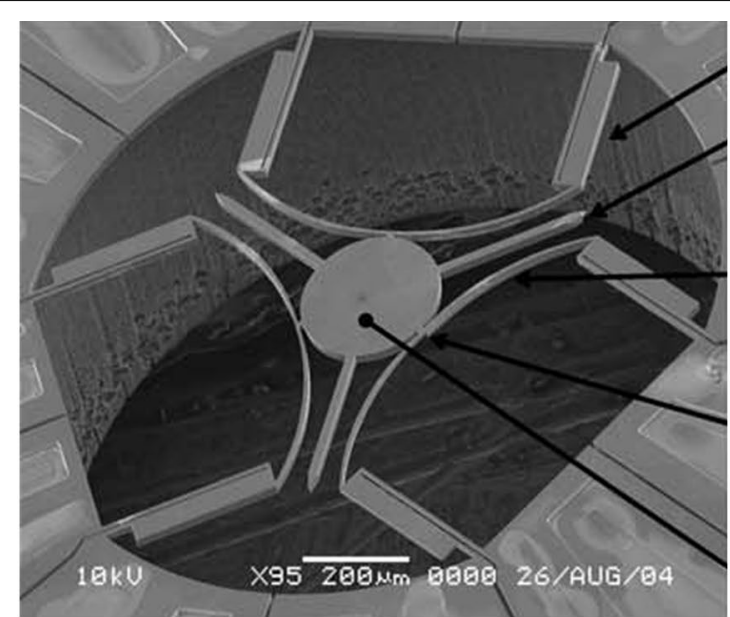

(a)

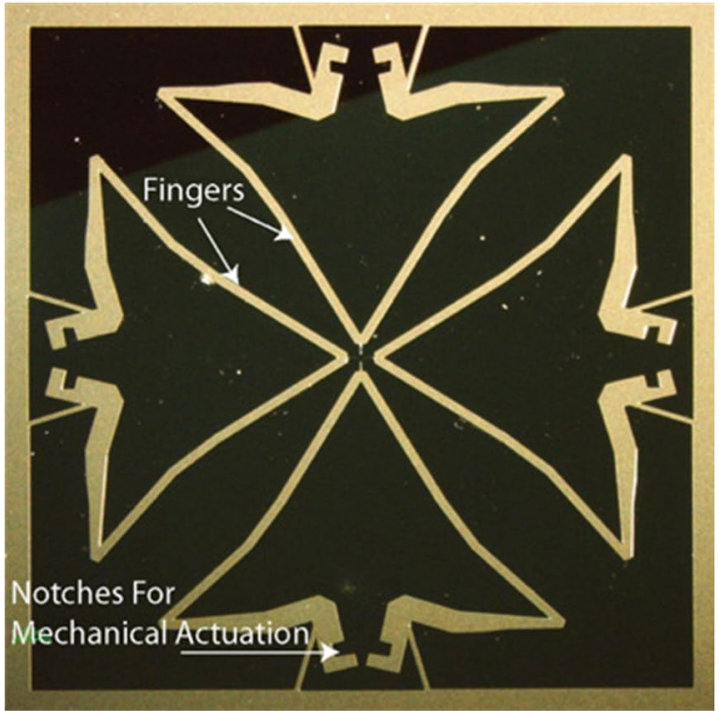

(c)

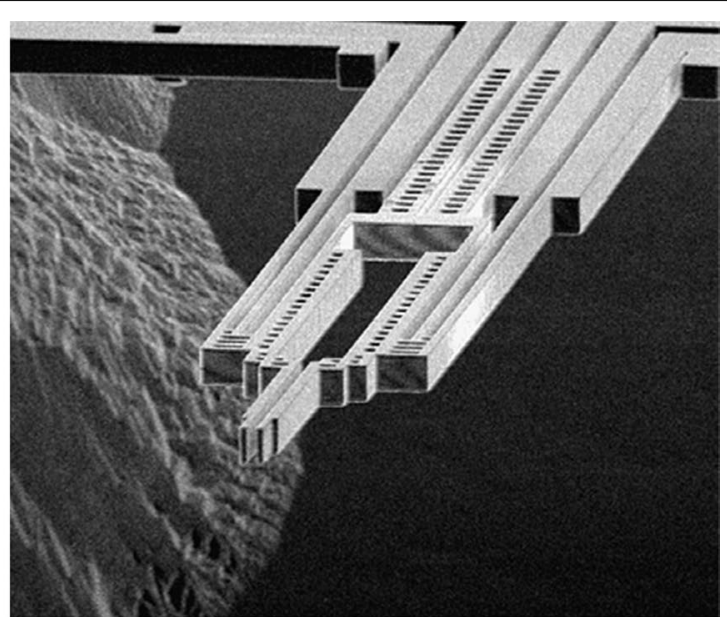

(b)

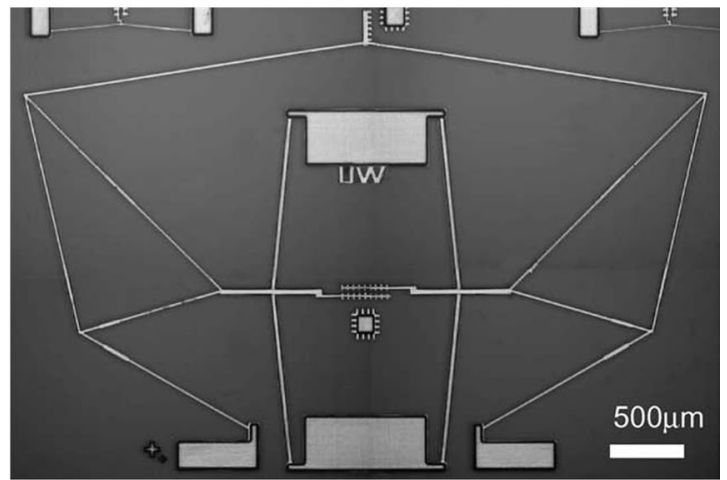

(d)

Figure 3 CMMs fabricated via bulk micromachining: (a) Nanopositioner by Chen and Culpepper [28] (@Elsevier. Adapted by permission of Elsevier. Permission to reuse must be obtained from the rightsholder), (b) Electrothermally actuated microgripper by Volland et al. [55] (@Elsevier. Adapted by permission of Elsevier. Permission to reuse must be obtained from the rightsholder), (c) Multi-fingered micromechanism for coordinated micro/ nano manipulation by Krishnan and Saggere [56] (OIOP. Adapted by permission of IOP. Permission to reuse must be obtained from the rightsholder), (d) Compliant microtransmission from the dissolved silicon wafer process by Chu et al. [57] (@Elsevier. Adapted by permission of Elsevier. Permission to reuse must be obtained from the rightsholder) 
$\mathrm{CO}_{2}$ and Nd:YAG lasers are used for ablation by direct writing on the material surface, whereas an excimer laser is used to process some polymers by projection through a mask. The process features show considerable variance with respect to different materials and laser systems.

Microgrippers account for a large percentage of all CMMs fabricated using laser technology (Figure 4). Alogla et al. fabricated a microgripper by cutting a PMMA sheet with a $\mathrm{CO}_{2}$ laser [64]. $\mathrm{CO}_{2}$ lasers are typically used to cut non-metal materials. Sheet metals and ceramics are commonly processed using an Nd:YAG laser, for which the smallest feature resolution of $\sim 20 \mu \mathrm{m}$ can be realized. For instance, Bordatchev and Nikumb fabricated a microgripper by cutting nickel foil, whereas Grossard et al. successfully cut a PZT (PIC151) plate [62, 65]. Given that the output of the excimer laser is usually projected onto the material surface through a predefined mask, the fabricated size can be reduced by a specific factor using an optical lens. Thus, a much higher resolution can be achieved. For instance, Chang et al. fabricated a microgripper by cutting a polyurethane (PU) film with an excimer laser [63]. During the projection, the fabricated size was reduced by a factor of $10 \times$, and a fabrication resolution of $10 \mu \mathrm{m}$ was realized.

\subsubsection{Wire Electrical Discharge Machining (EDM)}

Electrical discharge machining (EDM) is a manufacturing process in which a desired shape is obtained using electrical discharge, that is, sparks. EDM is commonly used to cut metals. Wire EDM is a particular process that uses a wire as a tool. Normally, a minimum resolution of 0.1-0.2 $\mathrm{mm}$ can be achieved, enabling the realization of CMMs with submillimeter feature sizes. The mechanisms that are fabricated via wire EDM usually have a large overall size of several millimeters. The materials involved are widely used metals, such as aluminum, titanium, and spring steel $[66,67]$. However, the thicknesses of the metal plates are much larger than those used in laser ablation. For instance, an aluminum plate with a thickness of $\sim 2 \mathrm{~mm}$ was used by Zubir and Schirinzadeh to fabricate microgrippers with a large deformation, as shown in Figure 5(a), by exploiting the relatively large flexibility allowed [68]. In addition, Miller et al. fabricated a variable-focus reflector using a $1 \mathrm{~mm}$ thick titanium plate, as shown in Figure 5(b) [69].

\subsubsection{Micromolding}

Micromolding facilitates the fabrication of microstructures by filling the structural materials into predefined molds. According to the filling method that is used, they can be categorized into microinjection molding, microcasting molding, and electroplating molding. Given that LIGA uses polymethylmethacrylate (PMMA) molds defined by X-ray photolithography, it is also included as a special type of micromolding. On a microscale, molds can be fabricated using photolithography, laser cutting, wire EDM, or bulk micromachining. Micromolding exhibits high flexibility in feasible structural materials, which include various kinds of polymers, metals, metal alloys, and even ceramics. However, not all these materials have been used for micromechanisms; to date, the materials used in molded micromechanisms mainly include nickel, NiFe alloy, PDMS, and 3Y-TZP ceramics. The micromechanisms of these materials will be reviewed in the following section.

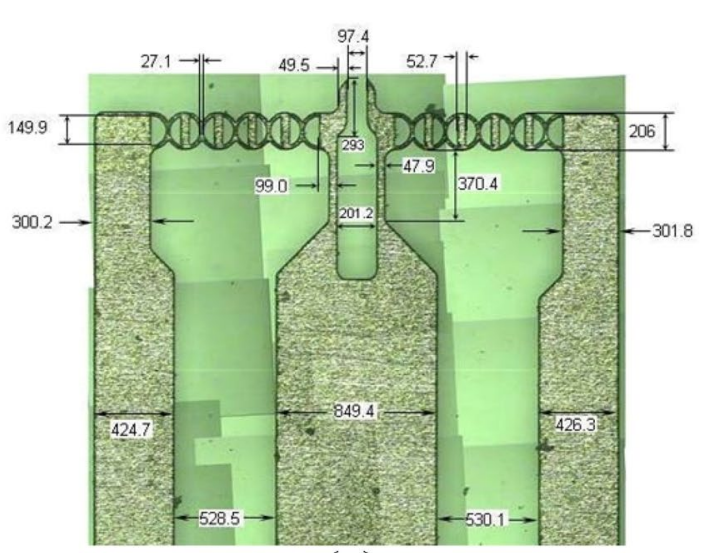

(a)

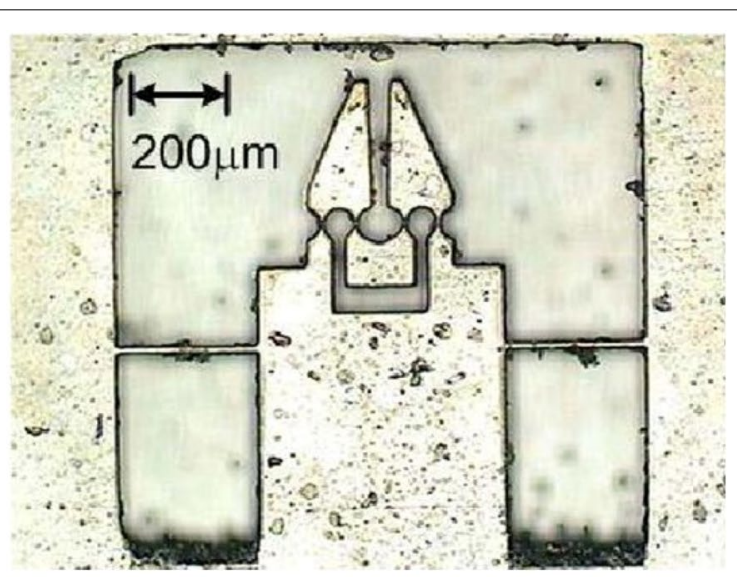

(b)

Figure 4 Microgrippers machined using laser technology: (a) Microgripper fabricated using nickel foil [62] (OIEEE. Adapted by permission of IEEE. Permission to reuse must be obtained from the rightsholder), (b) Microgripper fabricating using PU film [63] (@IEEE. Adapted by permission of IEEE. Permission to reuse must be obtained from the rightsholder) 


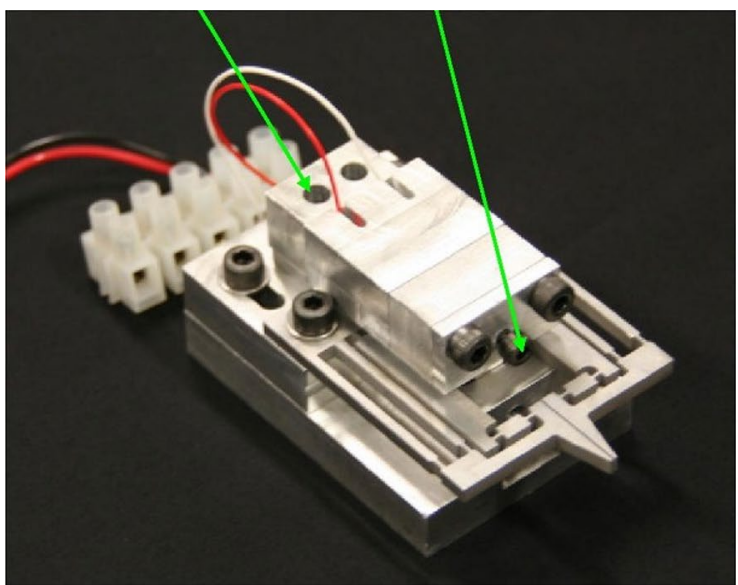

(a)

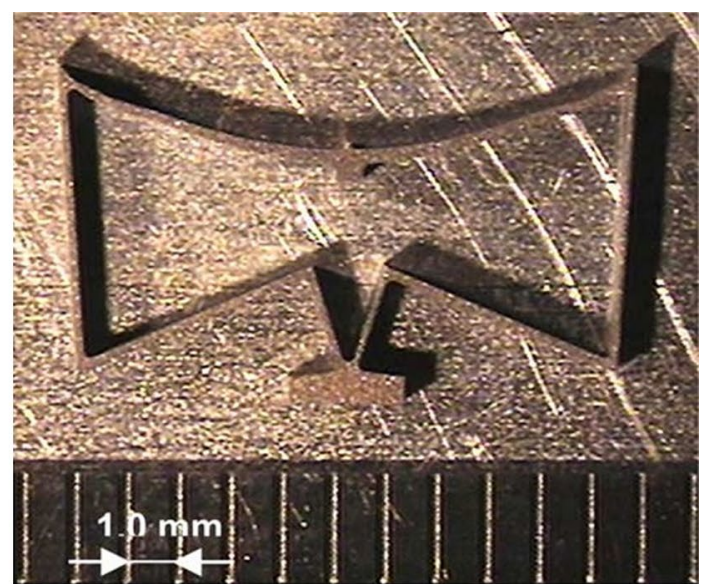

(b)

Figure 5 CMMs fabricated via wire EDM: (a) microgripper fabricated using Al plate [68] (OIEEE. Adapted by permission of IEEE. Permission to reuse must be obtained from the rightsholder), (b) variable-focus reflector fabricated using Ti plate [69] (@Elsevier. Adapted by permission of Elsevier. Permission to reuse must be obtained from the rightsholder)

Electroplated nickel and NiFe alloys are widely used in the realization of CMMs (Figure 6). The processes involved include LIGA and ordinary electroplating molding. LIGA, which is a German acronym for lithography, electroplating, and molding, was first realized in Germany as a milestone technique for the fabrication of high-aspect-ratio microstructures. Generally, the LIGA process involves two steps: defining the mold on thick PMMA using X-ray lithography and electroplating nickel into the predefined mold. LIGA can achieve a high aspect ratio (as high as 30 ) and considerable precision (less than $0.1 \mu \mathrm{m}$ per $100 \mu \mathrm{m}$ height), but it is very expensive because of the need for a synchrotron radiation source [70]. The reported CMMs made from LIGA include compliant microtransmission by Chu et al. and the microgripper by Carrozza, et al. [57, 71].

In addition to their application in LIGA, nickel and its alloys are also used in ordinary electroplating micromolding. The molds are usually defined by patterning photoresists, such as AZ4620, JSR, or SU-8 [73-75]. Given that these resists are exposed to UV light, this method is also referred to as UV LIGA. The structure thickness to be achieved usually depends on the resist. For instance, AZ4620 is approximately $20 \mu \mathrm{m}$ thick, whereas SU-8 facilitates a thickness up to $200 \mu \mathrm{m}$. It is worth noting that this method has been developed into MetalMUMPs by MEMSCAP, which serves as a standard process for $20 \mu \mathrm{m}$ thick nickel [76]. Although ordinary

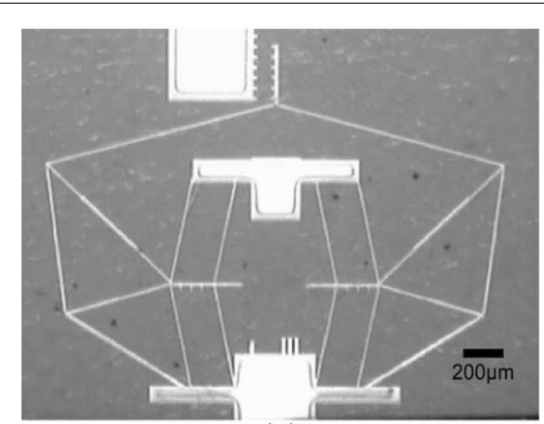

(a)

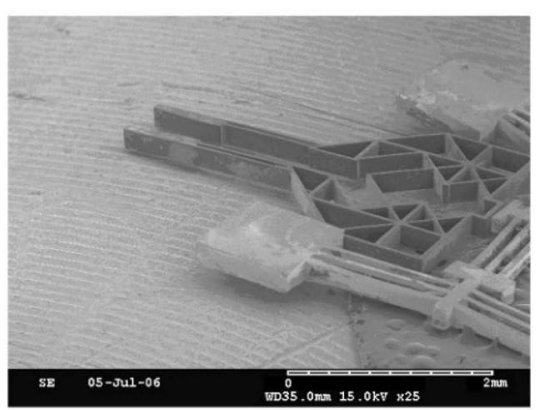

(b)

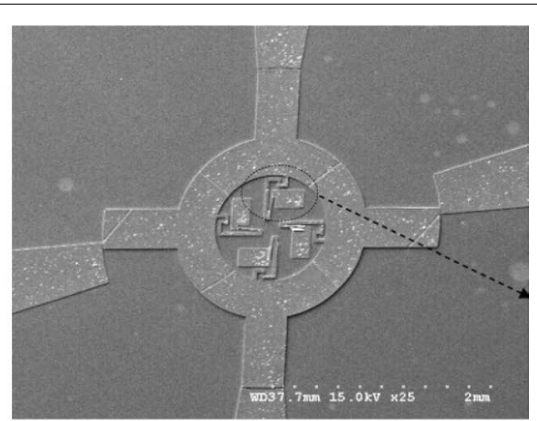

(c)

Figure 6 CMMs fabricated via electroplated Ni or NiFe: (a) Electrothermally-actuated compliant microtransmission fabricated using LIGA [57] (○Elsevier. Adapted by permission of Elsevier. Permission to reuse must be obtained from the rightsholder), (b) Microgripper fabricated from nickel, by Huang and Chen [72] (OIEEE. Adapted by permission of IEEE. Permission to reuse must be obtained from the rightsholder), (c) Compliant in-plane rotary bistable micromechanism fabricated from NiFe, by Luharuka and Hesketh [73] (@Elsevier. Adapted by permission of Elsevier. Permission to reuse must be obtained from the rightsholder) 
electroplating molding cannot achieve comparable accuracy to LIGA, it offers some advantages in terms of cost and flexibility. For all electroplate-related processes, the mechanical properties of either $\mathrm{Ni}$ or NiFe show considerable instability, and are strongly dependent on the electroplating conditions [77]. With respect to integrated actuation, numerous types of actuators are suitable, such as thermal actuators, electromagnetic actuators, and piezoelectric actuators [78].

Other widely used structural materials include polymers, such as polydimethylsiloxane (PDMS) and polyformaldehyde (POM), which are normally cast into a predefined mold (Figure 7). Molds are usually defined by silicon processing or electrical discharge micromachining (EDM) $[4,25,79]$. PDMS or POM is then poured into the mold, cured at a specific temperature, and finally ejected out of the mold. The mechanisms cast with different types of molds exhibit considerable differences in the smallest feature resolution. The silicon molds can usually create a minimum line width of several microns, whereas the EDM molds have a limit of several hundred microns. The greatest advantage of PDMS is its relatively low Young's modulus and high flexibility. If plastic deformation can be accepted, PDMS can be strained in excess of $200 \%$ before fracture [4]. In combination with a Young's modulus of only $\sim 1 \mathrm{MPa}$, it is suitable for the fabrication of high-sensitivity force sensors, energy-efficient microgrippers, and mechanisms that are folded out of the plane [4]. Moreover, its biocompatibility makes it an excellent candidate for bio-operations, medical devices, and even bionic robots.

Recently, researchers at the Pennsylvania State University presented a molding method called lost mold rapid infiltration forming (LM-RIF) that facilitates the fabrication of CMMs from ceramics or metals. The detailed steps of the process are illustrated in Figure 8 [80]. The molds were fabricated by patterning SU-8, which allows a high aspect ratio. Particulate ceramic or metal materials are cast into molds in the form of a colloidal suspension. Finally, mold removal and sintering were achieved in a single thermal treatment. To date, the reported materials for use include 3Y-TZP ceramics and particulate stainless steel 316L [80]. These materials have high Young's modulus and reliable failure stress, as well as considerable chemical inertness; therefore, this method has been used in the fabrication of contact-aided CMMs and surgical microgrippers [81-83].

\subsubsection{SU-8 Process}

SU-8 was introduced by IBM in 1989 as an epoxy material used in MEMS to replace the expensive X-ray lithography process in the LIGA technique. As a negative thick-film photoresist, SU-8 allows a high aspect ratio via photolithography and, therefore, facilitates mold preparation in micromolding. In addition to its application in micromolding, SU-8 is widely used as a structural material for CMMs by employing its mechanical properties. The typical SU-8 microfabrication process for CMMs is as follows: (1) A thin layer of copper or aluminum is sputtered on the substrate as the sacrificial layer and patterned if needed; (2) The SU-8 photoresist is spin-coated and exposed; (3) The exposed SU-8 is cured at a specific temperature to perform the cross-linking reaction; (4) The structure is formed by developing and then released by removing the sacrificial layer. This process enables the fabrication of CMMs requiring a thick structure layer and high aspect ratio at a much lower cost and time than the silicon process and micromolding.

Cured SU-8 has glass-like mechanical properties and a wide elastic range without plastic deformation owing

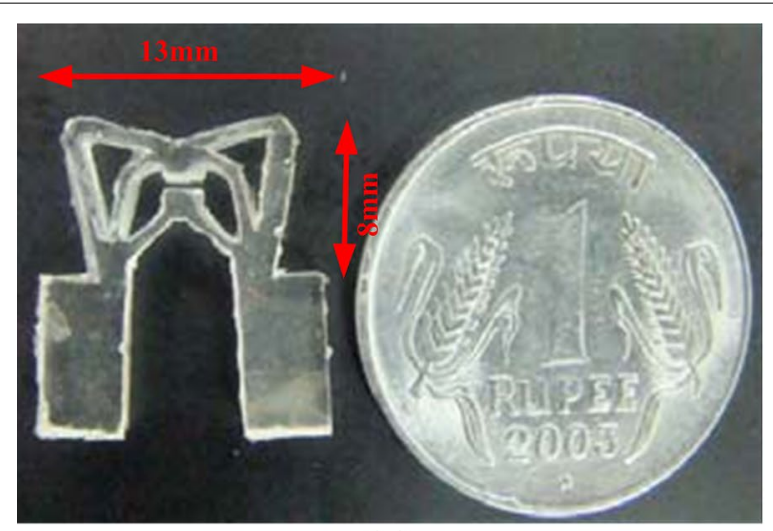

(a)

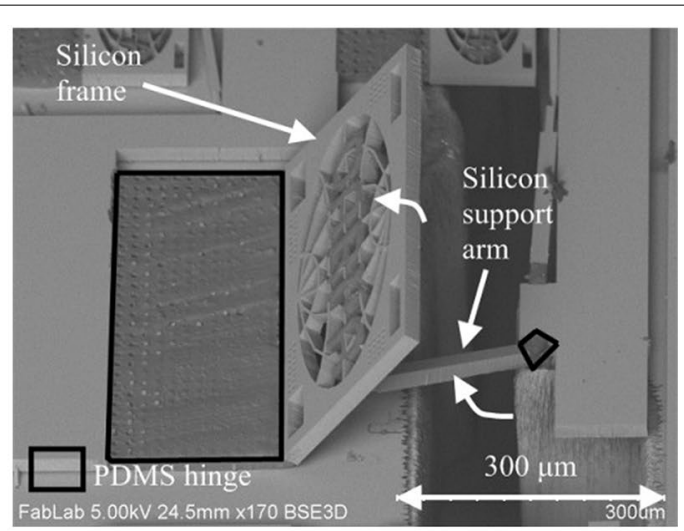

(b)

Figure 7 CMMs fabricated from PDMS: (a) Microgripper by Maheshwari et al. [84], (b) A silicon-PDMS hybrid out-of-plane mechanism with PDMS hinge by Gerratt et al. [4], (CIEEE. Adapted by permission of IEEE. Permission to reuse must be obtained from the rightsholder) 


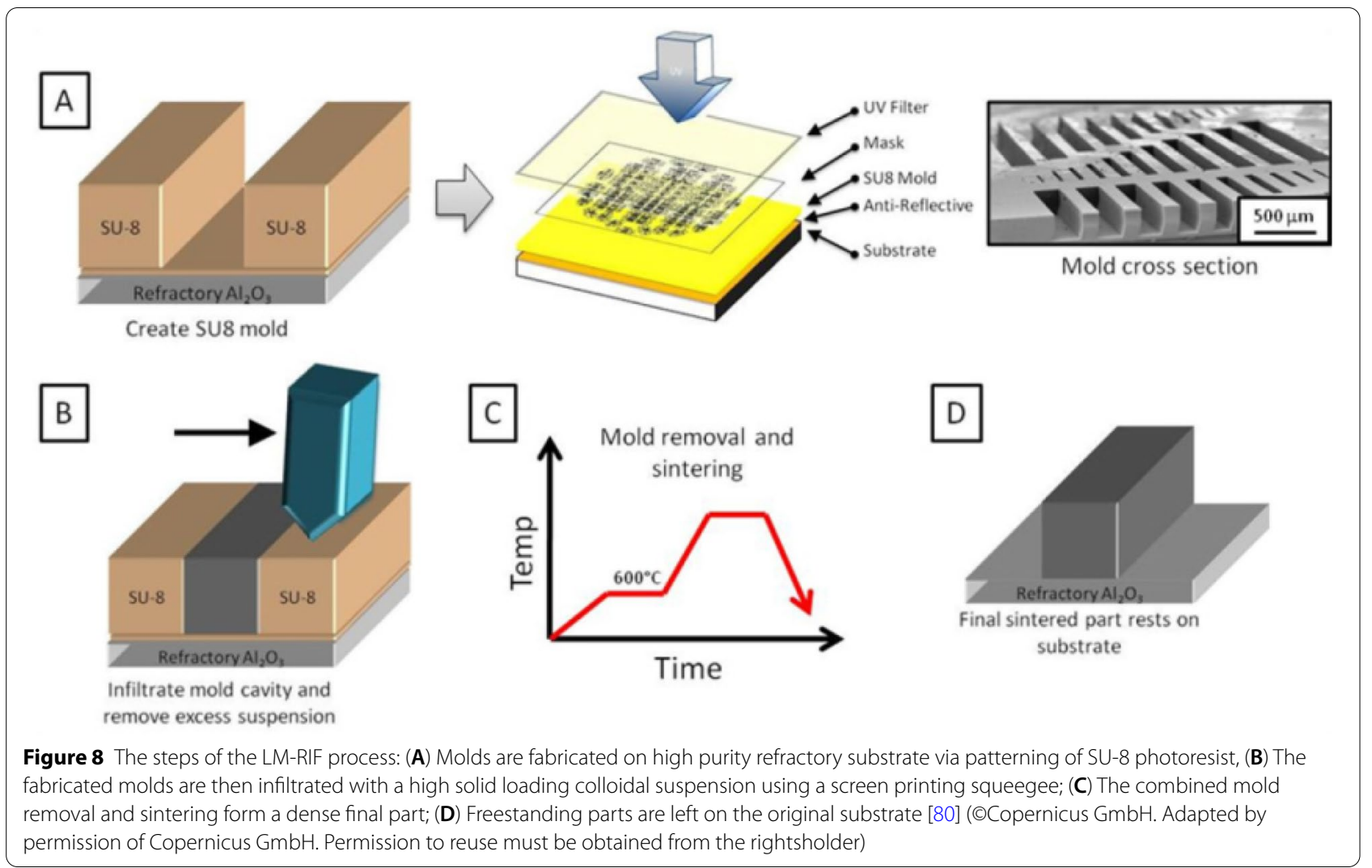

to the presence of a highly cross-linked matrix of covalent bonds. Given the low Young's modulus (2-5 GPa), SU-8 mechanisms can be much softer than those made from silicon or metal, and thus decrease the actuation force required. In addition, thin SU-8 can be used in out-of-plane mechanisms owing to its large flexibility (Figure 9) [44]. From the perspective of biocompatibility, SU-8 is suitable for the fabrication of microgrippers used in biological tissue and cell operations. Moreover, SU-8 is also a good choice to mimic the structure of insect wings because of its similar density and Young's modulus [85]. The SU8 process exhibits easy integration with different types of external actuators, such as piezoelectric actuators, shape memory alloys (SMAs), and pneumatic actuators [5, 86, 87]. Most importantly, the high thermal expansion coefficient of SU-8 (52 ppm/K) compared to silicon and metals allows the realization of monolithically integrated thermal actuation with moderate voltages [88].

\subsubsection{CNT Frameworking}

In recent years, the rapid development of nanotechnology has offered new opportunities for innovation in microfabrication. Some nanomaterials exhibit frameworking capabilities owing to their porous nature. As a result, different types of structural materials can be incorporated into the framework or nanomaterial mold. Such frameworking methods either result in nanocomposites with enhanced properties or introduce new desired material characteristics. For instance, Balucani et al. used patterned porous silicon as a framework and obtained nanocomposite materials with magnetic properties [89]. In the fabrication of CMMs, the most prospective frameworking material is carbon nanotubes (CNTs). Their composite allows for much more favorable mechanical properties than conventional MEMS materials.

Carbon nanotubes are the strongest and stiffest known materials [90]. The advent of the CNT forest technique, that is, the formation of vertically aligned CNT arrays, enables the CNT frameworking by bridging the dimensional gap between nanotubes and micromechanisms [91]. In commonly used catalytic chemical vapor deposition (CCVD), the CNT forest grows on a pre-patterned catalyst layer (e.g., Fe) by pyrolyzing feedstock gas [90]. The direction of growth of the CNT forest is usually identified as longitudinal, whereas the direction in the substrate surface is transverse. Remarkably, in the longitudinal direction, such CNT forests allow yield strains of $\sim 20 \%$, which allows a large deflection for CMMs [90]. Given that the binding strength between adjacent CNTs, that is, in the transverse direction, is too weak to 


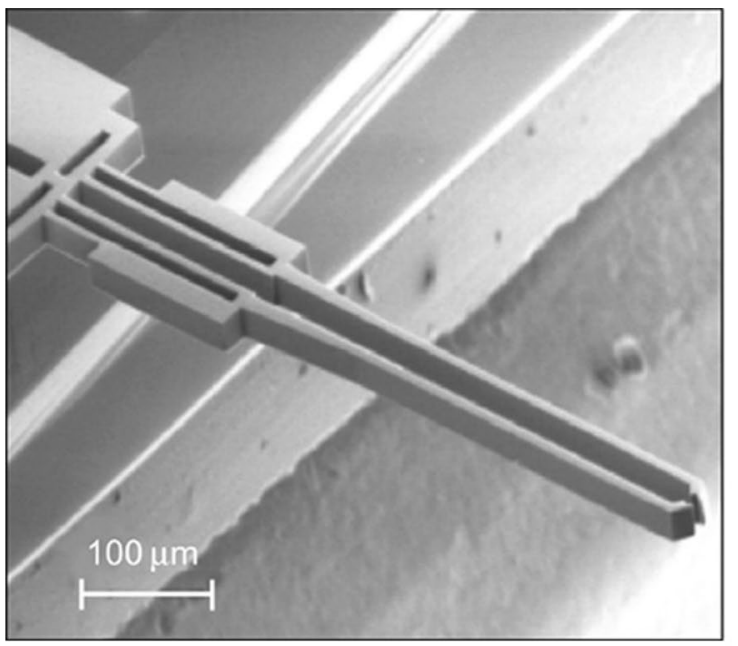

(a)

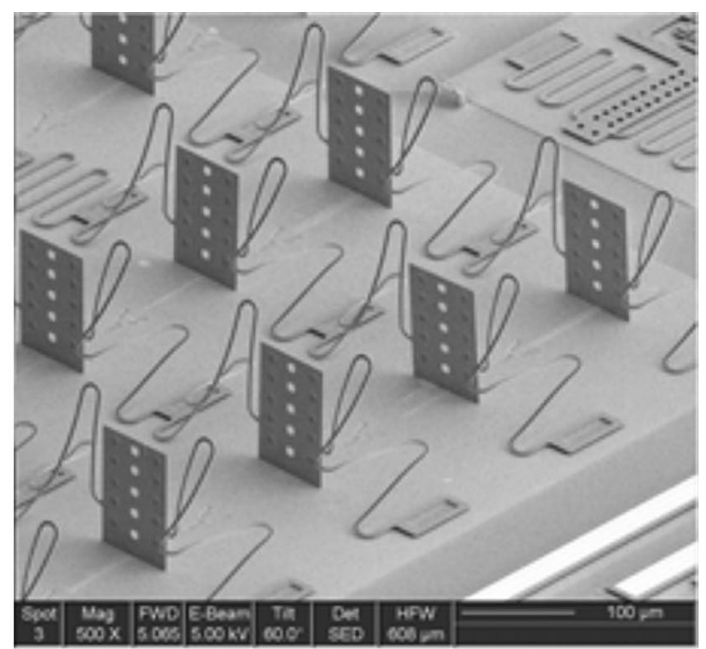

(b)

Figure 9 CMMs fabricated using SU-8: (a) Microgripper with integrated thermal actuator [88] (@IEEE. Adapted by permission of IEEE. Permission to reuse must be obtained from the rightsholder), (b) $90^{\circ}$ folded Tsang mechanism [44] (CIOP. Adapted by permission of IOP. Permission to reuse must be obtained from the rightsholder)

accommodate a large external load, the CNT framework is infiltrated with a filler material, resulting in a CNT composite. The existence of the CNT framework significantly modifies the mechanical properties of the composite. For example, the Young's modulus of the CNT-PU composite is 3.4 times larger than that of pure PU, as reported by Shin et al. [92].

The greatest advantage of such frameworks is that they facilitate versatile filler materials. Initially, the infiltration of several organic materials was investigated, for example, PDMS, paralene-C, and SU-8, wherein the methods used were vacuum casting, spin coating, capillary wetting, or chemical vapor deposition [91, 95]. These organic materials create CNT composites with low Young's modulus and large maximum allowable strain. However, the aspect ratio and fabrication accuracy are limited because of the high viscosity of the filler material and the large contamination caused by the infiltration method. In recent years, researchers from Brigham Young University (BYU) started to use atomistic CVD methods (e.g., LPCVD and ALD) to infiltrate the CNT framework with certain ceramics and metals such as polysilicon, amorphous carbon, molybdenum, tungsten, silicon nitride, silicon carbide, etc. [33, 96-98]. Benefitting from the conformal coating nature of the infiltration method, such a CNT frameworking facilitates a considerably large aspect ratio of at least 20, even 40 if the process is fully optimized. Moreover, both the cleanliness and fabrication accuracy are highly improved, because only a thin infiltration layer with a thickness comparable to the space between adjacent CNTs is needed. As reported, the fabrication deviation of the CNT frameworking is several times less than that of DRIE, and the cost is considerably lower than that of silicon processing. In the following two paragraphs, PDMS-infiltrated and carbon-infiltrated CNT frameworks, as well as the resultant material properties, are presented in detail.

PDMS is the most widely used organic filler material. Typically, CNT-PDMS composites are formed by casting PDMS into an as-defined CNT framework, which limits the maximum height of the CNT forest, which is usually less than $100 \mu \mathrm{m}$ [95]. Owing to the large flexibility of PDMS, the resultant composite is suitable for the fabrication of CMMs. It is worth noting that the mechanical properties of CNT composites usually exhibit anisotropic features. The longitudinal modulus of the resultant composite is significantly enhanced, with a maximum value of $\sim 18.87 \mathrm{MPa}$, whereas pure PDMS and CNT forest have values of $\sim 2.63 \mathrm{MPa}$ and $\sim 0.55 \mathrm{MPa}$, respectively [99]. The transverse modulus is $7.95 \mathrm{MPa}$, and Poisson's ratio is 0.2 [95]. The composite had a maximum strain of at least $20 \%$ before failure. Although the material properties have been investigated, well-designed CMMs made from CNT-PDMS composites have not been reported.

In all the studies related to the research performed at BYU, carbon-infiltrated CNT frameworks are investigated the most and have been used in the fabrication of different kinds of CMMs [33]. Amorphous carbon is infiltrated into the as-defined CNT framework by LPCVD [33]. The resultant composite has a Young's modulus of 4-6 GPa and a Poisson's ratio of $\sim 0.28$. In terms of flexibility, it can achieve a maximum strain of 
$2-2.5 \%$ before failure, notably larger than that of silicon, SU-8, and almost all engineering metals. It is quite impressive that many of the fabricated cantilevers experienced deflections greater than $10 \%$ of their length during testing [33]. This process has been utilized in the fabrication of micrograspers, compliant rotational hinges, lamina emergent mechanisms (LEMs), and constant force micromechanisms (Figure 10) [2, 33, 93, 94, 100, 101]. All of the aforementioned CMMs benefit from the low Young's modulus and large flexibility. For instance, the lamina emergent torsional joints fabricated by W. C. Fazio can achieve out-of-plane torsions of $60^{\circ}$ to $135^{\circ}$ without failure [33]. This enlarges the deflection of flexible members to achieve the desired mechanical motions or accomplishes the same motions with a higher safety factor.

\subsubsection{D Printing}

Three-dimensional (3D) printing has been widely used for both prototyping and the rapid production of CMMs. This technique can be divided into different categories based on the working principles. Fused deposition modeling (FDM) [102-104], micro continuous liquid interface production ( $\mu$ CLIP) [105], stereo lithography appearance (SLA) [106-109], and two-photon polymerization (2PP) [110] have been reported in the literature. 3D printing typically involves the use of polymers to fabricate CMMs. However, these printing methods demonstrate

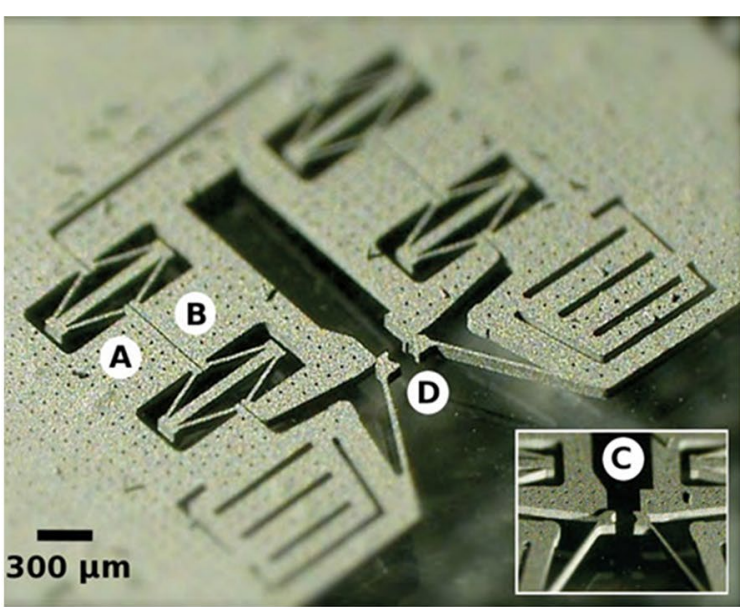

(a)
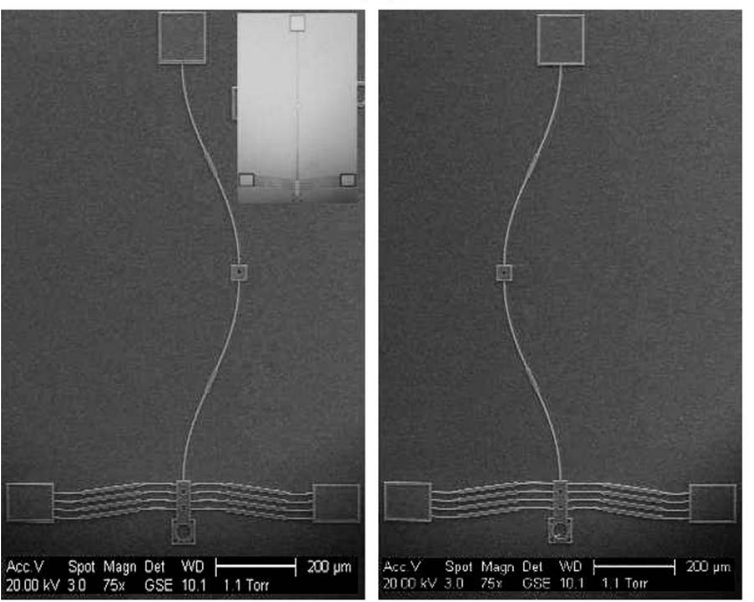

(c)

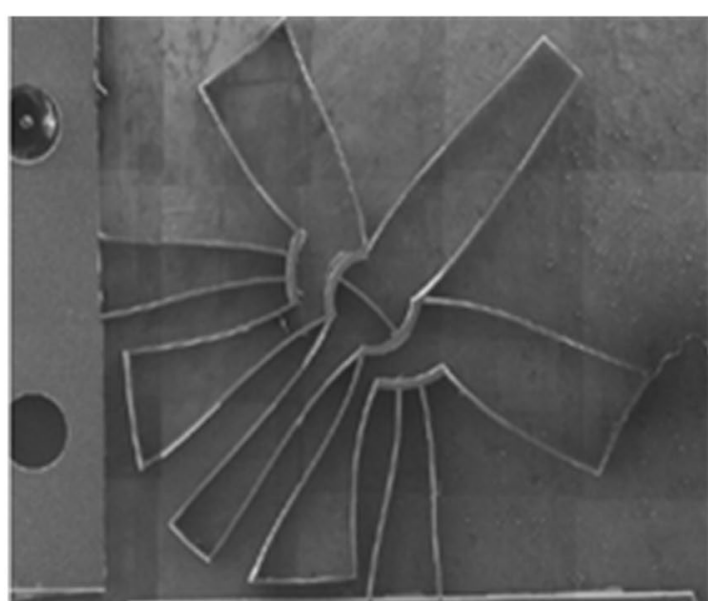

(b)

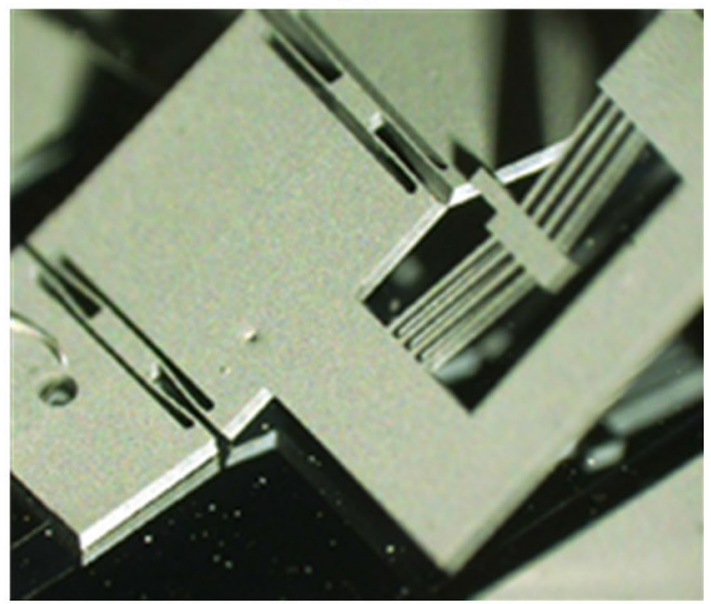

(d)

Figure 10 CMMs fabricated via CNT frameworking: (a) Micrograsper by Toone et al. [2] (๑Taylor\&Francis. Adapted by permission of Taylor\&Francis. Permission to reuse must be obtained from the rightsholder), (b) Compliant rotational hinge by Fowler et al. [93] (@Elsevier. Adapted by permission of Elsevier. Permission to reuse must be obtained from the rightsholder), (c) Tristable mechanism by Chen et al. [94] (๑ASME. Adapted by permission of ASME. Permission to reuse must be obtained from the rightsholder), (d) Lamina emergent mechanism by Fazio [33] 
very different manufacturing capabilities in terms of the smallest resolution and manufacturing error.

FDM works by extruding thermoplastic filaments through a heated nozzle, melting the material, and applying the plastic layer by layer to a build platform. Typically, the polymers used in FDM are acrylonitrile butadiene styrene (ABS), poly(lactic acid) (PLA), and polyamide (nylon). For example, Zirbel et al. fabricated bistable mechanisms using ABS as the prototyping material of FDM for potential space applications [102]; Almeida et al. used the FDM of nylon to fabricate robotic microtweezers [103]. FDM is a good choice for the prototyping of some preliminary designs of CMMs owing to the time and cost efficiency, but the available feature resolution is relatively large, commonly at the sub-millimeter level. FFF also shows tremendous advantages for rapid prototyping, but has limitations owing to the insufficient feature resolution in the realization of CMMs [104].

Photopolymer-based 3D printing technologies, such as stereo lithography (SLA), micro continuous liquid interface production ( $\mu$ CLIP), and two-photon polymerization (2PP) can be used to fabricate CMMs with $\mu \mathrm{m}$-level feature resolution. SLA is a form of 3D printing technology, in which light causes the photopolymer to crosslink and form the body of a 3D solid. The photopolymers reported in the literature include well-established photo-curable materials such as trichlorofluoromethane (R11) [106], photo-curable polymer mixtures [107], and special photopolymer resins developed by $3 \mathrm{D}$ printing companies such as Formlabs [108]. The smallest feature resolution that can be reliably achieved by SLA ranges from $20 \mu \mathrm{m}$ to $70 \mu \mathrm{m}$, as shown in Figure 11(a) and (b). If even smaller feature resolution is required, two-photon polymerization (2PP) can be applied to the fabrication of CMMs. 2PP technology can reduce the smallest feature resolution to $0.28-1.5 \mu \mathrm{m}$ [111], but the fabrication process is more sophisticated. 2PP technology requires a tightly focused laser beam on a defined volume of the photopolymer to enable the material to absorb two photons simultaneously to achieve an excited state. For such second-order processes, photon absorption is proportional to the square of the light intensity; therefore, crosslinking only occurs in a very small focused volume of the laser. As a result, 2PP technology can realize a feature resolution much smaller than SLA. For example, $M$. Power fabricated a monolithic force-sensitive 3D microgripper on the tip of a $125-\mu \mathrm{m}$-diameter optical fiber using two-photon polymerization of IP-Dip photoresist, achieving the smallest feature resolution of $\sim 1 \mu \mathrm{m}$ [110]. Continuous liquid interface production (CLIP) is also based on the polymerization of photopolymers activated by a light source, but the polymerization takes place only at the liquid/solid interface that is created by an oxygen-permeable window. In CLIP the objects continuously "grow" out of a pool of photopolymer rather than via layer by layer stacking, thereby, addressing some drawbacks of SLA and 2PP in terms of low efficiency. $\mu$ CLIP can achieve the smallest feature resolution of several microns. For example, Shao et al. fabricated a magnetically actuated microgripper using $\mu$ CLIP of a composite photopolymer of CN982A75 (Sartomer Inc.) and poly(ethylene glycol) diacrylate (PEGDA) (SigmaAldrich Inc.) with the addition of magnetic nanoparticles, which has a spatial resolution of $7.58 \times 7.58 \mu \mathrm{m}^{2}$.

\subsection{Results and discussions}

The process features and material properties were extracted from the literature and are listed in Table 4. It is evident that the manufacturing processes show a large variation in the smallest feature resolution and aspect ratio, both of which have substantial effects on the processing capability. The smallest feature resolution is closely related to the technique. Normally, photolithography-based processes have a much lower resolution compared to non-photolithographic techniques. On the other hand, the aspect ratio determines the ratio between the in-plane and out-of-plane stiffnesses of the fabricated mechanisms. In many cases, a high aspect ratio is desirable. For instance, a high aspect ratio is usually required for microgrippers to realize high out-of-plane stiffnesses and ensure the safe transportation of micro-objects. Among all the processes, bulk micromachining, LIGA, SU-8, CNT frameworking, and 3D printing allow an aspect ratio greater than 20 . As previously indicated, the need for constituent materials with a low Young's moduli and high maximum allowable strains is increasing, to fabricate more sophisticated CMMs. To meet this need, various types of constituent materials have been introduced in the fabrication of compliant motion amplifiers, LEMs, microgrippers, etc. Generally, polymers and composites demonstrate a lower Young's modulus and a larger maximum allowable strain than silicon.

To provide further assistance to designers, the process features and material properties are interpreted with respect to the fabrication of a particular kind of CMM. The process features are determined by the process itself and the material involved. For this reason, in the following paragraphs, the interpretation is performed with respect to process-material combinations. Thirteen process-material combinations were selected from Table 4, based on their utilization frequency and information completeness. These process-material combinations were as follows: (1) Surface-micromachining-polysilicon; (2) Bulk-micromachining-SCS; (3) Laser-ablation$\mathrm{Al}$; (4) Laser-ablation-Ni; (5) Laser-ablation-PMMA; (6) EDM-Al; (7) EDM-Ti; (8) LIGA-Ni; (9) Molding-Ni; (10) 


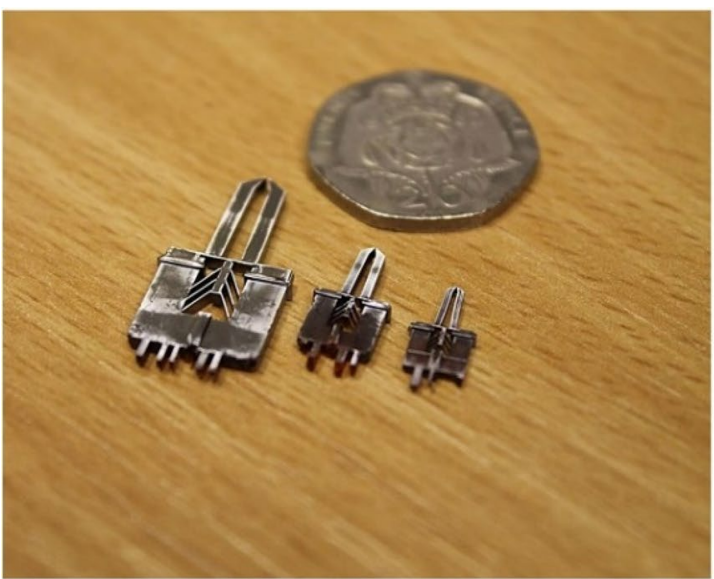

(a)

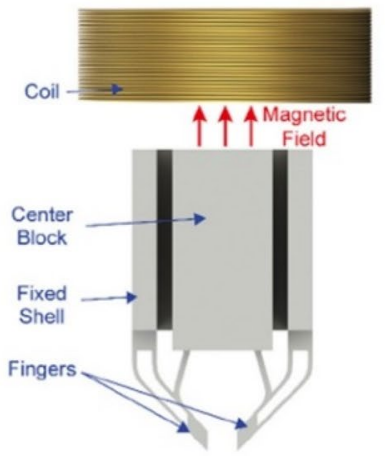

(c)
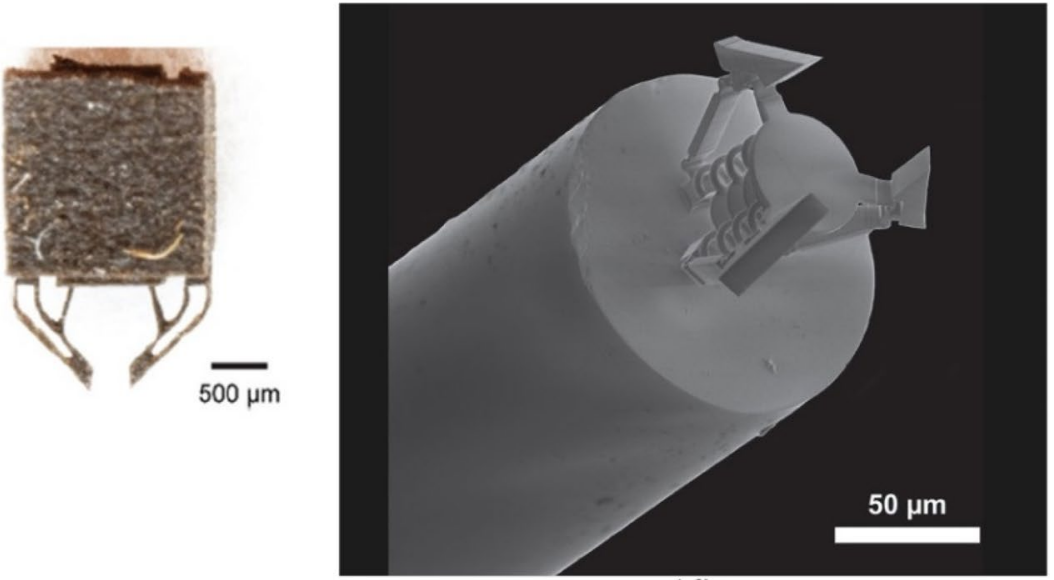

(d)

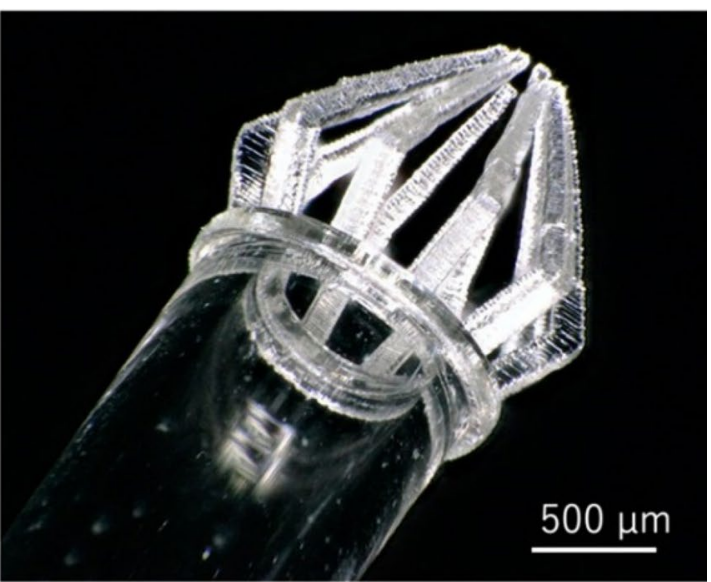

(b)

Figure 11 CMMs fabricated using 3D printing: (a) Microgripper by K. Alblalaihid, et al. with SLA of R11 photopolymer [106]. (OIOP. Adapted by permission of IOP. Permission to reuse must be obtained from the rightsholder.) (b) Micromanipulator by S. Kozaki, et al. with SLA of a mixed photo-curable polymer [107]. (@MDPI. Adapted by permission of MDPI. Permission to reuse must be obtained from the rightsholder.) (c) Magnetically-actuated microgripper by G. Shao using MCLIP [105]. (@Elsevier. Adapted by permission of Elsevier. Permission to reuse must be obtained from the rightsholder.) (d) Monolithic force-sensitive 3D microgripper by M. Power with 2PP [110]. (OWiley. Adapted by permission of Wiley. Permission to reuse must be obtained from the rightsholder.)

SU-8; (11) CNT-frameworking-carbon; (12). SLA-R11; (13) 2PP-IP-Dip. The CMMs are planar structures in most cases, except those fabricated via 3D printing. The geometry is confined by the process capability, especially the smallest feature resolution and aspect ratio. Eventually, the geometry affects the performance of CMMs, in conjunction with the material properties. For compliant mechanisms, Young's modulus and the maximum allowable strain are the material properties that are of greatest concern to designers. For this reason, the smallest feature resolution, aspect ratio range, Young's modulus, and maximum allowable strain of the process-material combinations are shown explicitly in a graphical format. In a further step, the appropriateness of the process and material suitability are considered with respect to a particular kind of CMM.

The graph of the smallest feature resolution vs. aspect ratio range is shown in Figure 12. Based on the smallest feature resolution, the 13 combinations were ranked as follows: LIGA-Ni $<2$ PP-IP-Dip < surface-micromachining-polysilicon $<$ bulk-micromachining-SCS $<$ molding$\mathrm{Ni}<\mathrm{CNT}$-frameworking-carbon $<$ SU-8 $<$ laser-ablatio$\mathrm{n}$-Al $<$ laser-ablation-Ni $<$ SLA-R11 $<$ EDM-Ti $<$ EDM$\mathrm{Al}<$ laser-ablation-PMMA. This is consistent with the technological basis. Laser ablation and wire EDM are based on macroscale machining. Even though they are also used to fabricate compliant mechanisms with microscale components, their smallest feature resolution is 


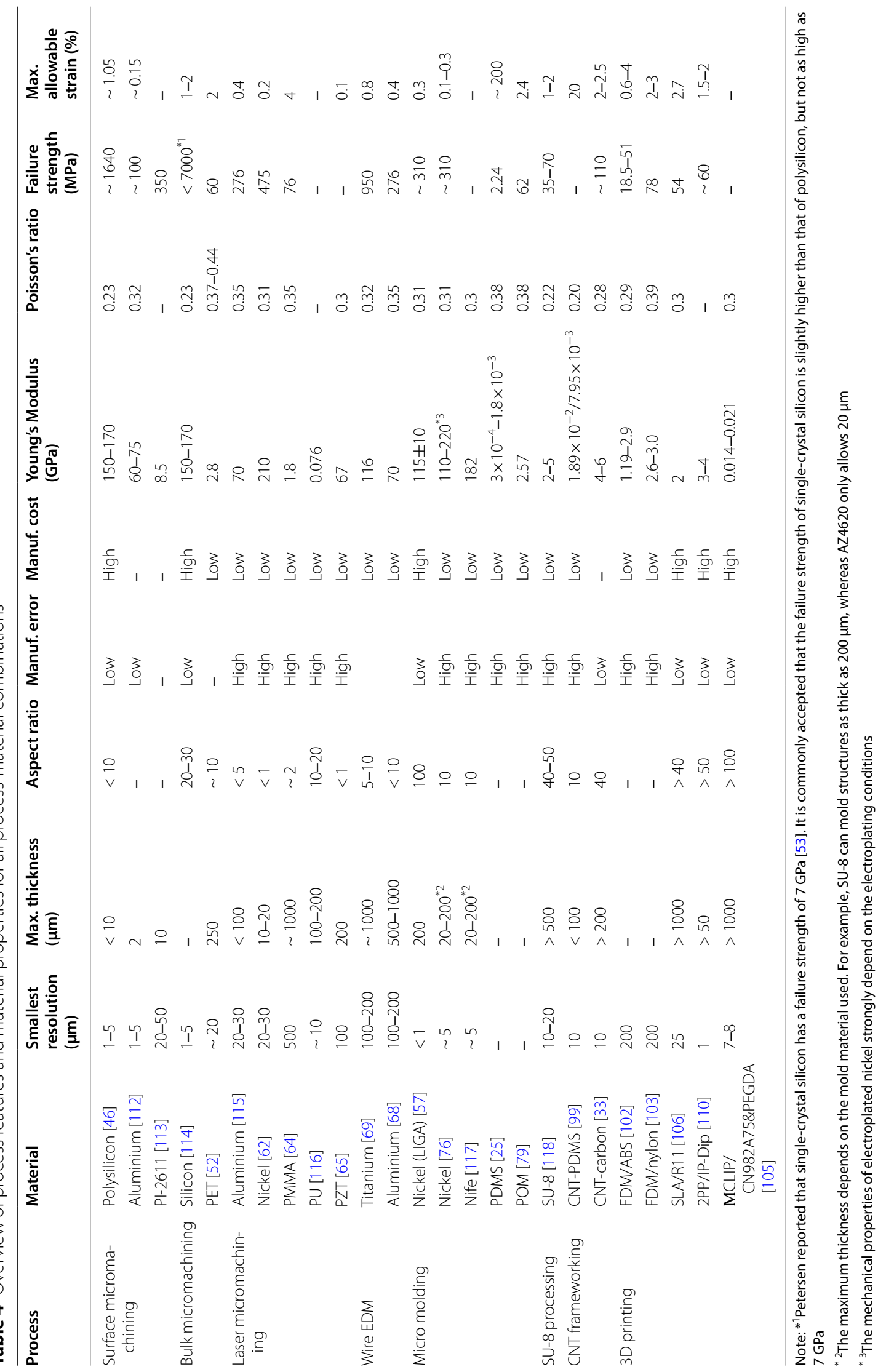




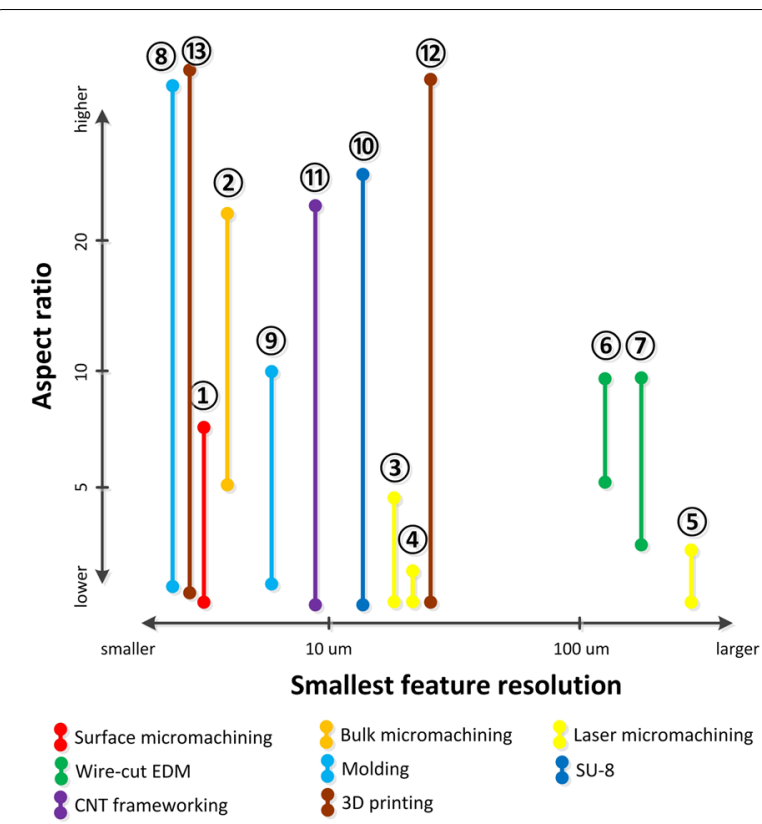

Figure 12 Graph of smallest feature resolution vs. aspect ratio range depending on the process material

relatively larger than that of other photolithographybased processes. Therefore, these processes based on photolithography are much more advanced than laser ablation and wire EDM from the perspective of smaller feature size and batch processing capability.

In addition to the smallest feature resolution, the aspect ratio is another key parameter for evaluating microfabrication processes. The aspect ratio ranges of these processes are shown along the $y$-axis. Generally, this parameter determines the ratio between the in-plane and orthogonal stiffness for particular planar flexures. Some mechanisms with in-plane motion only require a large aspect ratio to increase the orthogonal stiffness and enhance the robustness. For example, a microgripper requires a high aspect ratio to reliably perform a grasping task. Among all the process-material combinations, LIGA-Ni, bulk-micromachining-SCS, CNT-frameworking-carbon, SU-8, SLA-R11, and 2PP-IP-Dip allow aspect ratios larger than 20 . Therefore, these processes are frequently used to fabricate microgrippers, whereas others, such as surface-micromachining-polysilicon and laser ablation are inappropriate because of the relatively low aspect ratio that can be achieved.

On the contrary, some CMMs fulfill their function depending on the out-of-plane motion; therefore, a large aspect ratio is undesirable for such mechanisms. A prominent example is lamina emergent mechanisms (LEMs) [44], which depend on a thin structure layer to achieve out-of-plane motion. Currently, reported LEMs are made

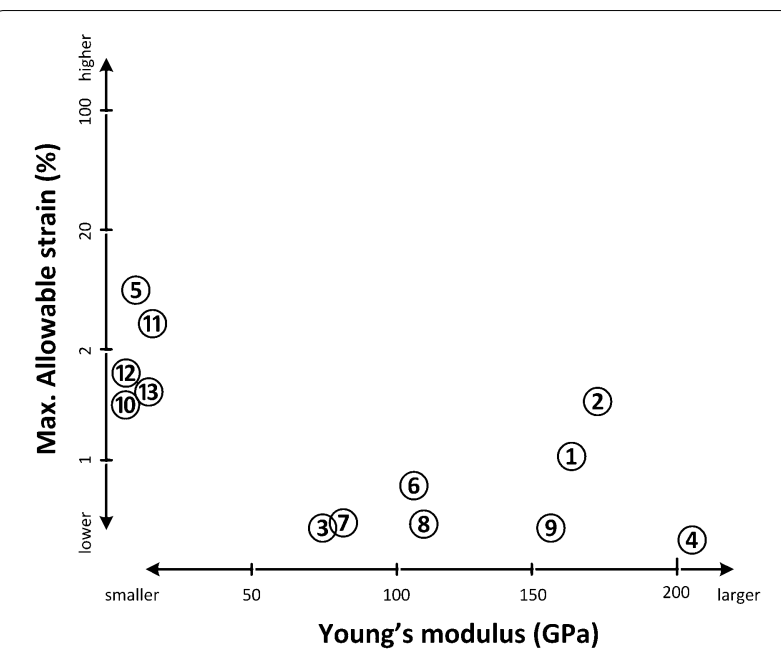

Figure 13 Graph of Young's modulus vs. maximum allowable strain of the materials in the 13 process-material combinations

from surface-micromachining-polysilicon, SU-8, and CNT-frameworking-carbon. If a small feature size is not required, laser ablation is also a good choice for obtaining LEMs from metal foil. However, some of the combinations, for example, bulk-micromachining-SCS, are difficult to fabricate because the thickness of crystalline silicon is normally very large, even if SOI wafers are used.

From the perspective of material properties, a low Young's modulus and large maximum allowable strain are highly favored in the realization of compliant mechanisms. Softer materials result in lower stiffness and less actuation effort. In addition, a large maximum allowable strain means that the mechanisms can deflect to a much higher degree; therefore, the performance can be significantly enhanced. The materials of the 13 processmaterial combinations are shown in the graph of Young's modulus vs. maximum allowable strain in Figure 13. In general, these combinations can be divided into two groups. One group is located on the bottom-right side of the graph, indicating a higher Young's modulus and lower maximum allowable strain. This group includes both silicon and conventional metals. In contrast, laser-ablationPMMA, SU-8, CNT-frameworking-carbon, SLA-R11, and 2PP-IP-Dip are on the top-left side of the graph. Therefore, PMMA, SU-8, CNT-carbon composites, and some photopolymers are more suitable for the fabrication of CMMs.

To further compare their suitability, the maximum allowable strain of the materials in the 13 process-material combinations are plotted, as shown in Figure 14. As the most widely used material in microfabrication, polysilicon has a maximum allowable strain of $\sim 1 \%$, which is larger than that of conventional metals. SCS has a 


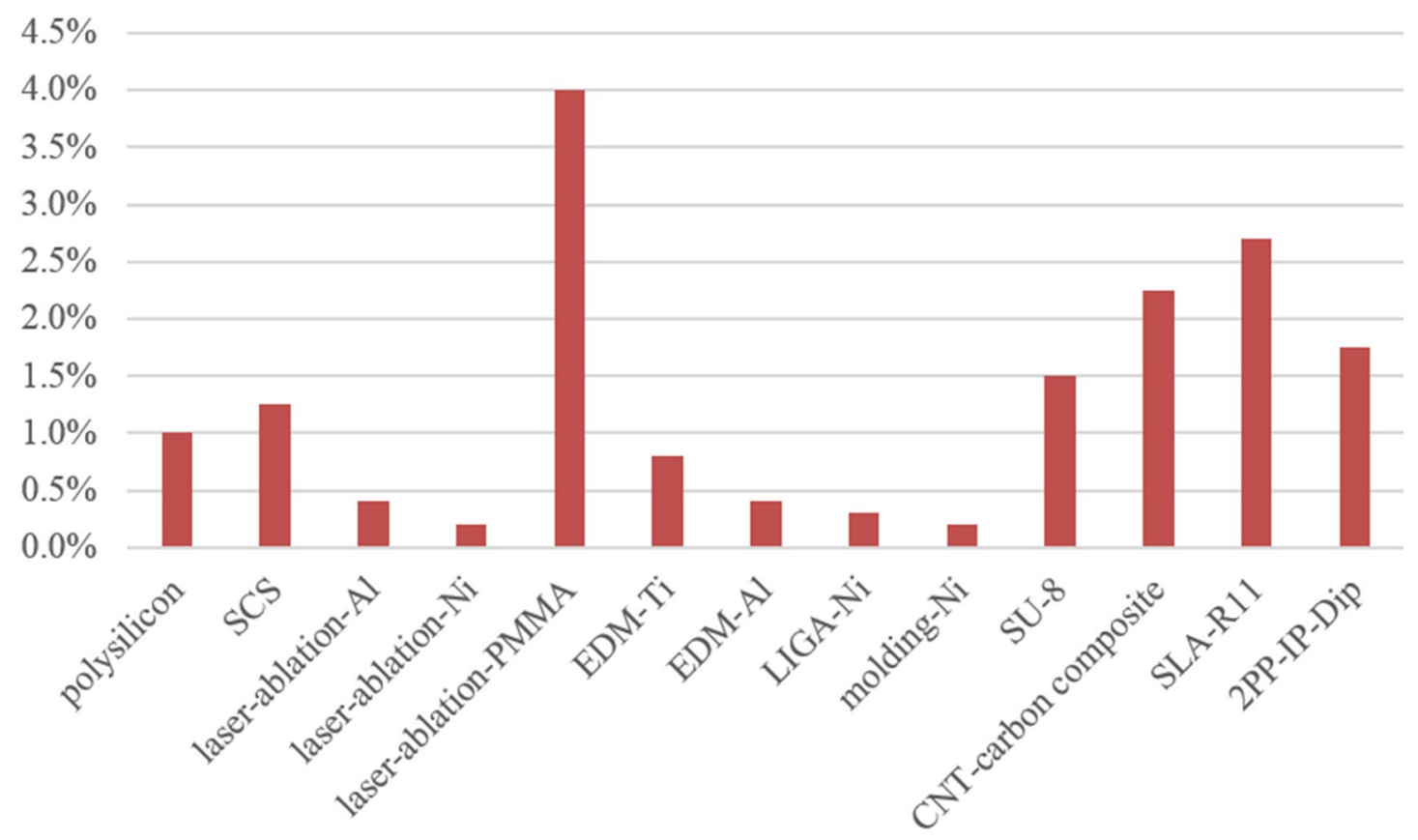

Figure 14 Column chart of the maximum allowable strain of the 13 process-material combinations

maximum allowable strain of $1.25 \%$, which is slightly larger than that of polysilicon. PMMA, SU-8, CNT-carbon composite, R11, and IP-Dip allow a larger maximum strain than SCS. It is worth noting that PMMA has only been processed by laser ablation, which is limited to a feature resolution of $0.5 \mathrm{~mm}$, and causes considerable fabrication deviation, even though its maximum allowable strain is as high as $4 \%$. Therefore, apart from silicon, CNT-carbon composites and some photopolymers such as SU8, R11, and IP-Dip are excellent materials for the fabrication of CMMs.

\section{Conclusions}

In this report, the fabrication of compliant micromechanisms is reviewed and discussed with respect to the process features and material properties. The literature surveyed was classified and statistically analysed. The state-of-the-art of CMM fabrication with representative processes was summarized. The process features and material properties were extracted from the literature and compiled into an information table as a reference for designers. Finally, the process-material combinations were characterized using graphs, and guidelines for the selection of manufacturing processes, and constituent materials were discussed with respect to different kinds of CMMs.
The development of CMM fabrication generally comes along with MEMS. As a result, silicon is the most widely used method. However, several polymer and composite processes are being rapidly developing, and significant progress has been achieved. These new processes and the associated materials have some advantages compared to the silicon process in terms of the Young's modulus, maximum allowable strain, aspect ratio, and manufacturing cost. These advantages are highly favored, as CMMs are become increasingly sophisticated and require large deflections. Moreover, as the utilization of CMMs expands to biotechnology, bionics, and robotics, these polymers and composites have more advantages over silicon owing to their biocompatibility.

The smallest feature resolution and aspect ratio are two critical aspects in the selection of an appropriate manufacturing process. The smallest feature resolution of a particular process is determined not only by the inherent limitations of the technology, but also by the processed material. In general, lithography-based processes have smaller feature sizes than processes derived from macro-scale machining. It was determined that some types of CMMs have specific requirements in terms of the aspect ratio, which impose limitations in the selection of the process and the material. For instance, bulk micromachining, SU-8, CNT frameworking, and 3D printing are highly favored by 
microgrippers, which require a large aspect ratio; however, the reported LEMs were only fabricated using surface micromachining, the SU-8 process, and the CNT frameworking, in which a very thin structural layer can be achieved.

A low Young's modulus and a large maximum allowable strain are highly favored in the realization of compliant mechanisms. It was determined that the constituent materials that are currently used in CMMs can be roughly divided into two groups, considering their Young's modulus and maximum allowable strain. Generally, Young's moduli of silicon and conventional metals are relatively high and do not allow a large maximum strain. In contrast, Young's moduli of polymers and composite materials are much lower, and their maximum allowable strains are much larger; therefore, they are more suitable for the fabrication of CMMs. Considering the process features and material properties, it is assumed that the CNT frameworking and some photopolymer-based processes, such as SU-8, SLA, and 2PP, are potential candidates for the fabrication of future high-performance CMMs.

\section{Acknowledgements}

The authors would like to thank Patrice Lambert and Davood F. Machekposhti of Delft University of Technology for the kind help for review paper writing.

\section{Authors' contributions}

MW surveyed the literature and wrote the manuscript; DG made additional literature survey on EDM and micro molding; LZ assisted in data collection and diagram drawing; JLH assisted in modifying the structure and content of the paper. All authors read and approved the final manuscript.

\section{Authors' Information}

Minchang Wang, born in 1987, is currently a researcher at Institute of Intelligent Flexible Mechatronics, Jiangsu University, China. He received his PhD degree and bachelor degree from Shanghai Institute of Microsystem and Information Technology, Chinese Academy of Sciences, in 2014 and Nanjing University, China, in 2009, respectively. His research interests include flexible sensors, MEMS sensors and sensor-based intelligent systems.

Daohan Ge, born in 1982, is currently an associate professor at School of Mechanical Engineering, Jiangsu University, China. She received her PhD degree from Shanghai Institute of Microsystem and Information Technology, Chinese Academy of Sciences, in 2010.

Liqiang Zhang, born in 1984, is currently an associate professor at School of Mechanical Engineering, Jiangsu University, China. He received his PhD degree from Jiangsu University, China, in 2012.

Just L. Herder is a full professor of Interactive Mechanisms and Mechatronics, Chair of the Mechatronic System Design Group and Head of Department of Precision and Microsystems Engineering, Delft University of Technology, The Netherlands. His research interests focus on mechatronic systems with remarkable behavior and their applications.

\section{Funding}

Supported by Jiangsu University Foundation (Grant No. 20JDG37).

\section{Competing Interests}

The authors declare no competing financial interests.

\section{Author Details}

${ }^{1}$ Institute of Intelligent Flexible Mechatronics, Jiangsu University, Zhenjiang 212013, China. ${ }^{2}$ Department of Precision and Microsystems Engineering, Faculty of Mechanical, Maritime and Materials Engineering, Delft University of Technology, Mekelweg 2, 2628CD Delft, The Netherlands.

Received: 16 June 2020 Revised: 19 May 2021 Accepted: 19 August 2021 Published online: 08 September 2021

\section{References}

[1] R P Feynman. There's plenty of room at the bottom. California Institute of Technology, Engineering and Science Magazine, 1960.

[2] N C Toone, W C Fazio, J M Lund, et al. Investigation of unique carbon nanotube cell restraint compliant mechanisms. Mechanics Based Design of Structures and Machines, 2014, 42(3): 343-354.

[3] S Ku, S Salcudean. Design and control of a teleoperated microgripper for microsurgery. Proceedings of IEEE International Conference on Robotics and Automation, 1996: 889-894.

[4] A P Gerratt, I Penskiy, S Bergbreiter. Integrated silicon-PDMS process for microrobot mechanisms. 2010 IEEE International Conference on Robotics and Automation, 2010: 3153-3158.

[5] V Seidemann, S Bütefisch, S Büttgenbach. Fabrication and investigation of in-plane compliant SU8 structures for MEMS and their application to micro valves and micro grippers. Sensors and Actuators A: Physical, 2002, 97: 457-461.

[6] G-W Kim, J Kim. Compliant bistable mechanism for low frequency vibration energy harvester inspired by auditory hair bundle structures. Smart Materials and Structures, 2012, 22(1): 014005.

[7] M Dinesh, G Ananthasuresh. A topology-optimized large-range compliant XY micro stage. 13th National Conference on Mechanisms and Machines (NaCoMM07), IISC, Bangalore, India, 2007.

[8] D Clements, L Howell, N Masters, et al. Floating pin joints fabricated from two layers of polysilicon at the micro level. Tenth World Conference on the Theory of Machines and Mechanisms, 1999: 20-24.

[9] R Yeh, E J Kruglick, K S Pister. Surface-micromachined components for articulated microrobots. Journal of Microelectromechanical Systems, 1996, 5(1): 10-17.

[10] A Friedberger, R S Muller. Improved surface-micromachined hinges for fold-out structures. Journal of Microelectromechanical Systems, 1998, 7(3): 315-319.

[11] J J Allen, H K Schriner. Micromachine wedge stepping motor. Sandia National Laboratories, Albuquerque, NM, and Livermore, CA, 1998.

[12] G Ananthasuresh, H Rothbart. Cams in microelectromechanical systems. Cam Design Handbook, 2004: 505-527.

[13] D B Asay, M T Dugger, S H Kim. In-situ vapor-phase lubrication of MEMS. Tribology Letters, 2008, 29(1): 67-74.

[14] R Maboudian, W R Ashurst, C Carraro. Self-assembled monolayers as anti-stiction coatings for MEMS: characteristics and recent developments. Sensors and Actuators A: Physical, 2000, 82(1-3): 219-223.

[15] A Ghosh, B Corves. Introduction to micromechanisms and microactuators. Springer, 2015.

[16] S Kota, J Hetrick, Z Li, et al. Tailoring unconventional actuators using compliant transmissions: design methods and applications. IEEE/ASME Transactions on Mechatronics, 1999, 4(4): 396-408.

[17] S Kota, J Joo, Z Li, et al. Design of compliant mechanisms: applications to MEMS. Analog Integrated Circuits and Signal Processing, 2001, 29(1): 7-15.

[18] M Wang, J Jiao, P Yan, et al. A novel tri-axis MEMS gyroscope with inplane tetra-pendulum proof masses and enhanced sensitive springs. Journal of Micromechanics and Microengineering, 2014, 24(4): 045002.

[19] S Kota, J Hetrick, Z Li, et al. Synthesizing high-performance compliant stroke amplification systems for MEMS. Proceedings IEEE Thirteenth Annual International Conference on Micro Electro Mechanical Systems (Cat. No. 00CH36308), 2000: 164-169.

[20] N D Masters, L L Howell. A self-retracting fully compliant bistable micromechanism. Journal of Microelectromechanical Systems, 2003, 12(3): 273-280. 
[21] D L Wilcox, L L Howell. Fully compliant tensural bistable micromechanisms (FTBM). Journal of Microelectromechanical Systems, 2005, 14(6): 1223-1235.

[22] $\mathrm{J} O \mathrm{~J}$ Jacobsen, G Chen, L L Howell, et al. Lamina emergent torsional (LET) joint. Mechanism and Machine Theory, 2009, 44(11): 2098-2109.

[23] QT Aten, B D Jensen, L L Howell. Geometrically non-linear analysis of thin-film compliant MEMS via shell and solid elements. Finite Elements in Analysis and Design, 2012, 49(1): 70-77.

[24] L Saggere, S Kota, S Crary. A new design for suspension of linear microactuators. ASME Journal of Dynamic Systems and Control, 1994, 55(2): 671-675.

[25] G S Baichapur, H Gugale, A Maheshwari, et al. A vision-based microNewton static force sensor using a displacement-amplifying compliant mechanism (DaCM). Mechanics Based Design of Structures and Machines, 2014, 42(2): 193-210.

[26] S Khan, G Ananthasuresh. Improving the sensitivity and bandwidth of in-plane capacitive microaccelerometers using compliant mechanical amplifiers. Journal of Microelectromechanical Systems, 2014, 23(4): 871-887.

[27] G Ananthasuresh, N Maheswari, A N Reddy, et al. Fabrication of spring steel and PDMS grippers for the micromanipulation of biological cells. In: Microfluidics and microfabrication. Springer. 2010: 333-354.

[28] S-C Chen, M L Culpepper. Design of a six-axis micro-scale nanopositioner- $\mu$ HexFlex. Precision Engineering, 2006, 30(3): 314-324.

[29] Y Li, Q Zheng, Y Hu, et al. Micromachined piezoresistive accelerometers based on an asymmetrically gapped cantilever. Journal of Microelectromechanical Systems, 2011, 20(1): 83-94.

[30] J-S Lee, E-S Yoo, C-H Park, et al. Development of a piezoresistive MEMS pressure sensor for a precision air data module. 2014 14th International Conference on Control, Automation and Systems (ICCAS 2014), 2014: 874-878.

[31] L C Leishman, D J Ricks, M B Colton. Design and evaluation of statically balanced compliant mechanisms for haptic interfaces. Dynamic Systems and Control Conference, 2010: 859-866.

[32] M C Carrozza, G Cappiello, G Stellin, et al. A cosmetic prosthetic hand with tendon driven under-actuated mechanism and compliant joints: ongoing research and preliminary results. Proceedings of the 2005 IEEE International Conference on Robotics and Automation, 2005: 2661-2666.

[33] W C Fazio. Mechanical properties and MEMS applications of carboninfiltrated carbon nanotube forests. Brigham Young University, 2012.

[34] C Liu. Foundations of MEMS. Pearson Education India, 2012.

[35] W D Callister, D G Rethwisch. Materials science and engineering: An introduction. Wiley New York, 2018.

[36] C Maier, T Calafut. Polypropylene: the definitive user's guide and databook. William Andrew, 1998.

[37] P Pal, K Sato, S Chandra. Fabrication techniques of convex corners in a (1 0 0)-silicon wafer using bulk micromachining: A review. Journal of Micromechanics and Microengineering, 2007, 17(10): R111.

[38] S Saadon, O Sidek. A review of vibration-based MEMS piezoelectric energy harvesters. Energy Conversion and Management, 2011, 52(1): 500-504.

[39] STadigadapa, K Mateti. Piezoelectric MEMS sensors: state-of-the-art and perspectives. Measurement Science and Technology, 2009, 20(9): 092001.

[40] J W Wittwer, M S Baker, L L Howell. Simulation, measurement, and asymmetric buckling of thermal microactuators. Sensors and Actuators A: Physical, 2006, 128(2): 395-401.

[41] D Galayko, A Kaiser, B Legrand, et al. Clamped-clamped beam micromechanical resonators in thick-film epitaxial polysilicon technology. 32nd European Solid-State Device Research Conference, 2002: 447-450

[42] QT Aten, B D Jensen, S H Burnett, et al. A self-reconfiguring metamorphic nanoinjector for injection into mouse zygotes. Review of Scientific Instruments, 2014, 85(5): 055005.

[43] M S Baker, L L Howell. On-chip actuation of an in-plane compliant bistable micromechanism. Journal of Microelectromechanical Systems, 2002, 11(5): 566-573.

[44] S-H Tsang, D Sameoto, I G Foulds, et al. Automated assembly of hingeless 90 out-of-plane microstructures. Journal of Micromechanics and Microengineering, 2007, 17(7): 1314.

[45] TMoulton, G Ananthasuresh. Micromechanical devices with embedded electro-thermal-compliant actuation. Sensors and Actuators A: Physical, 2001, 90(1-2): 38-48.
[46] J W Wittwer, M S Baker, L L Howell. Robust design and model validation of nonlinear compliant micromechanisms. Journal of Microelectromechanical Systems, 2006, 15(1): 33-41.

[47] S Bromley, L Howell, B Jensen. Determination of maximum allowable strain for polysilicon micro-devices. Engineering Failure Analysis, 1999, 6(1): 27-41.

[48] J Carter, A Cowen, B Hardy, et al. PolyMUMPs design handbook: revision 11.0. MEMSCAP Inc, 2005, 39.

[49] B D Jensen, L L Howell, L G Salmon. Introduction of two-link in-plane, bistable compliant MEMS. Proceeding of the 1998 ASME Design Engineering Technical Conferences, 1998.

[50] N Dechev, W L Cleghorn, J K Mills. Microassembly of 3-D microstructures using a compliant, passive microgripper. Journal of Microelectromechanical Systems, 2004, 13(2): 176-189.

[51] G Chen, D L Wilcox, L L Howell. Fully compliant double tensural tristable micromechanisms (DTTM). Journal of Micromechanics and Microengineering, 2009, 19(2): 025011.

[52] M Hajhashemi, F Barazandeh, S N Nejad, et al. Design and microfabrication of a constant-force microgripper. Proceedings of the Institution of Mechanical Engineers, Part C: Journal of Mechanical Engineering Science, 2011, 225(11): 2739-2748,

[53] KE Petersen. Silicon as a mechanical material. Proceedings of the IEEE, 1982, 70(5): 420-457.

[54] K-S Chen, A A Ayon, S M Spearing. Silicon strength testing for mesoscale structural applications. MRS Online Proceedings Library (OPL), 1998, 518.

[55] B Volland, K Ivanova, T Ivanov, et al. Duo-action electro thermal micro gripper. Microelectronic Engineering, 2007, 84(5-8): 1329-1332.

[56] S Krishnan, L Saggere. A multi-fingered micromechanism for coordinated micro/nano manipulation. Journal of Micromechanics and Microengineering, 2007, 17(3): 576

[57] L L Chu, J A Hetrick, Y B Gianchandani. High amplification compliant microtransmissions for rectilinear electrothermal actuators. Sensors and Actuators A: Physical, 2002, 97: 776-783.

[58] J Qiu, J H Lang, A H Slocum. A curved-beam bistable mechanism. Journal of Microelectromechanical Systems, 2004, 13(2): 137-146.

[59] P H Pham, L B Dang, DT Nguyen, et al. A micro transmission system based on combination of micro elastic structures and ratchet mechanism. Microsystem Technologies, 2017, 23(2): 381-387.

[60] H Du, F S Chau, G Zhou. Harmonically-driven snapping of a micromachined bistable mechanism with ultra-small actuation stroke. Journal of Microelectromechanical Systems, 2018, 27(1): 34-39.

[61] D F Machekposhti, J L Herder, G Sémon, et al. A compliant micro frequency quadrupler transmission utilizing singularity. Journal of Microelectromechanical Systems, 2018, 27(3): 506-512.

[62] EV Bordatchev, S Nikumb. Microgripper: design, finite element analysis and laser microfabrication. Proceedings International Conference on MEMS, NANO and Smart Systems, 2003: 308-313.

[63] R-J Chang, Y Lin, C Shiu, et al. Development of SMA-actuated microgripper in micro assembly applications. IECON 2007-33rd Annual Conference of the IEEE Industrial Electronics Society, 2007: 2886-2891.

[64] A Alogla, P Scanlan, W Shu, et al. A scalable syringe-actuated microgripper for biological manipulation. Sensors and Actuators A: Physical, 2013, 202: 135-139.

[65] M Grossard, C Rotinat-Libersa, N Chaillet, et al. Mechanical and controloriented design of a monolithic piezoelectric microgripper using a new topological optimization method. IEEE/ASME Transactions on Mechatronics, 2009, 14(1): 32-45.

[66] F Wang, C Liang, Y Tian, et al. Design and control of a compliant microgripper with a large amplification ratio for high-speed micro manipulation. IEEE/ASME Transactions on Mechatronics, 2016, 21 (3): 1262-1271.

[67] D Zhang, Z Zhang, Q Gao, et al. Development of a monolithic compliant SPCA-driven micro-gripper. Mechatronics, 2015, 25: 37-43.

[68] M N M Zubir, B Shirinzadeh. Development of a compliant-based microgripper for microassembly. 2008 IEEE/ASME International Conference on Mechtronic and Embedded Systems and Applications, 2008: 522-527.

[69] S F Miller, C-C Kao, A J Shih, et al. Investigation of wire electrical discharge machining of thin cross-sections and compliant mechanisms. International Journal of Machine Tools and Manufacture, 2005, 45(15): 1717-1725. 
[70] HGuckel.High-aspect-ratio micromachining via deepX-raylithography. Proceedings of the IEEE, 1998, 86(8): 1586-1593.

[71] M C Carrozza, P Dario, A Menciassi, et al. Manipulating biological and mechanical micro-objects using LIGA-microfabricated end-effectors. Proceedings. 1998 IEEE International Conference on Robotics and Automation (Cat. No. 98CH36146), 1998: 1811-1816.

[72] S-C Huang, W-L Chen. Design of topologically optimal microgripper. 2008 IEEE International Conference on Systems, Man and Cybernetics, 2008: 1694-1698.

[73] R Luharuka, P J Hesketh. Design of fully compliant, in-plane rotary, bistable micromechanisms for MEMS applications. Sensors and Actuators A: Physical, 2007, 134(1): 231-238.

[74] B-S Kim, J-S Park, B H Kang, et al. Fabrication and property analysis of a MEMS micro-gripper for robotic micro-manipulation. Robotics and Computer-Integrated Manufacturing, 2012, 28(1): 50-56.

[75] K Kim, E Nilsen, T Huang, et al. Metallic microgripper with SU-8 adaptor as end-effectors for heterogeneous micro/nano assembly applications. Microsystem Technologies, 2004, 10(10): 689-693.

[76] J J Khazaai, H Qu, M Shillor, et al. Design and fabrication of electrothermally activated micro gripper with large tip opening and holding force. SENSORS, 2011 IEEE, 2011: 1445-1448.

[77] T Fritz, M Griepentrog, W Mokwa, et al. Determination of Young's modulus of electroplated nickel. Electrochimica Acta, 2003, 48(20-22): 3029-3035.

[78] D-AWang, H-TPham, Y-H Hsieh. Dynamical switching of an electromagnetically driven compliant bistable mechanism. Sensors and Actuators A: Physical, 2009, 149(1): 143-151.

[79] W-B Kim, S-Y Han. Microinjection molding of out-of-plane bistable mechanisms. Micromachines, 2020, 11(2): 155.

[80] N E Antolino, G Hayes, R Kirkpatrick, et al. Lost mold rapid infiltration forming of mesoscale ceramics: Part 1, fabrication. Journal of the American Ceramic Society, 2009, 92: 563-569.

[81] V V Mehta. Design, analysis, and applications of cellular contact-aided compliant mechanisms. The Pennsylvania State University, 2010.

[82] M Aguirre. Design and optimization of narrow-gauged contact-aided compliant mechanisms for advanced minimally invasive surgery. The Pennsylvania State University, 2011.

[83] W Haouas, R Dahmouche, J Agnus, et al. New integrated siliconPDMS process for compliant micro-mechanisms. Journal of Micromechanics and Microengineering, 2017, 27(12): 127001.

[84] N Maheswari, A N Reddy, D K Sahu, et al. Miniature compliant grippers with force-sensing. 14th Nat. Conf. Mach. Mech., Durgapur, India, 2009.

[85] T Dargent, X Bao, S Grondel, et al. Micromachining of an SU-8 flapping-wing flying micro-electro-mechanical system. Journal of Micromechanics and Microengineering, 2009, 19(8): 085028.

[86] S-C Huang, G-J Lan. Design and fabrication of a micro-compliant amplifier with a topology optimal compliant mechanism integrated with a piezoelectric microactuator. Journal of Micromechanics and Microengineering, 2006, 16(3): 531.

[87] I Roch, P Bidaud, D Collard, et al. Fabrication and characterization of an SU-8 gripper actuated by a shape memory alloy thin film. Journal of Micromechanics and Microengineering, 2003, 13(2): 330.

[88] N Chronis, L P Lee. Electrothermally activated SU-8 microgripper for single cell manipulation in solution. Journal of Microelectromechanical Systems, 2005, 14(4): 857-863.

[89] M Balucani, N P Belfiore, R Crescenzi, et al. The development of a MEMS/NEMS-based 3 DOF compliant micro robot. 19th International Workshop on Robotics in Alpe-Adria-Danube Region (RAAD 2010), 2010: 173-179.

[90] A Cao, P L Dickrell, W G Sawyer, et al. Super-compressible foamlike carbon nanotube films. Science, 2005, 310(5752): 1307-1310.

[91] C-F Hu, C-M Lin, W Fang. Integration of Pdms-infiltrated CNTs and Si bulk-micromachining for monolithic physical sensors application. 2013 Transducers \& Eurosensors XXVII: The 17th International Conference on Solid-State Sensors, Actuators and Microsystems (TRANSDUCERS \& EUROSENSORS XXVII), 2013: 1565-1568.

[92] M K Shin, J Oh, M Lima, et al. Elastomeric conductive composites based on carbon nanotube forests. Advanced materials, 2010, 22(24): 2663-2667.

[93] RFowler, S Magleby, L Howell. Flex-16: A monolithic large-displacement compliant rotational hinge. Proceedings of IDETC/CIE, 2012.
[94] G Chen, QT Aten, S Zirbel, et al. A tristable mechanism configuration employing orthogonal compliant mechanisms. Journal of Mechanisms and Robotics, 2010, 2(1).

[95] G Keulemans, F Ceyssens, M De Volder, et al. Fabrication and characterisation of carbon nanotube composites for strain sensor applications. Proceedings of the 21st Micromechanics and Micro systems Europe Workshop, 2010: 231-234

[96] D N Hutchison, N B Morrill, Q Aten, et al. Carbon nanotubes as a framework for high-aspect-ratio MEMS fabrication. Journal of Microelectromechanical Systems, 2009, 19(1): 75-82.

[97] R Hansen. Mechanical and electrical properties of carbonnanotubetemplated metallic microstructures. Citeseer, 2012.

[98] RH Poelma, B Morana, SVollebregt, et al. Tailoring the mechanical properties of high-aspect-ratio carbon nanotube arrays using amorphous silicon carbide coatings. Advanced Functional Materials, 2014, 24(36): 5737-5744

[99] L Ci, J Suhr, V Pushparaj, et al. Continuous carbon nanotube reinforced composites. Nano Letters, 2008, 8(9): 2762-2766.

[100] N Tolou, P Pluimers, B D Jensen, et al. Constant force micro mechanism out of carbon nanotube forest. Proceedings of the 12th euspen International Conference Stockholm-Jun, 2011.

[101] L K Barrett, D J Barton, S G Noyce, et al. High-aspect-ratio metal microfabrication by nickel electroplating of patterned carbon nanotube forests. Journal of Microelectromechanical Systems, 2015, 24(5): 1331-1337.

[102] S A Zirbel, K A Tolman, B P Trease, et al. Bistable mechanisms for space applications. PloS One, 2016, 11(12): e0168218.

[103] A Almeida, G Andrews, D Jaiswal, et al. The actuation mechanism of 3D printed flexure-based robotic microtweezers. Micromachines, 2019, 10(7): 470.

[104] H M Anver, R Mutlu, G Alici. 3D printing of a thin-wall soft and monolithic gripper using fused filament fabrication. 2017 IEEE International Conference on Advanced Intelligent Mechatronics (AIM), 2017: 442-447.

[105] G Shao, H O T Ware, J Huang, et al. 3D printed magnetically-actuating micro-gripper operates in air and water. Additive Manufacturing, 2021, 38: 101834.

[106] K Alblalaihid, J Overton, S Lawes, et al. A 3D-printed polymer microgripper with self-defined electrical tracks and thermal actuator. Journal of Micromechanics and Microengineering, 2017, 27(4): 045019.

[107] S Kozaki, Y Moritoki, T Furukawa, et al. Additive manufacturing of micromanipulator mounted on a glass capillary for biological applications. Micromachines, 2020, 11(2): 174.

[108] L S Rogers, J C Van Wert, A F Mensinger. An implantable two axis micromanipulator made with a 3D printer for recording neural activity in free-swimming fish. Journal of Neuroscience Methods, 2017, 288: 29-33.

[109] M Power, G-Z Yang. Direct laser written passive micromanipulator end-effector for compliant object manipulation. 2015 IEEE/RSJ International Conference on Intelligent Robots and Systems (IROS), 2015: 790-797.

[110] M Power, A J Thompson, S Anastasova, et al. A monolithic force-sensitive 3D microgripper fabricated on the tip of an optical fiber using 2-photon polymerization. Small, 2018, 14(16): 1703964

[111] M Straub, M Gu. Near-infrared photonic crystals with higher-order bandgaps generated by two-photon photopolymerization. Optics Letters, 2002, 27(20): 1824-1826.

[112] S Pamidighantam, W Laureyn, C Rusu, et al. A wet release process for fabricating slender and compliant suspended micro-mechanical structures. Sensors and Actuators A: Physical, 2003, 103(1-2): 202-212.

[113] S-H Tsang, A H Ma, K S Karim, et al. Monolithically fabricated polymermems 3-axis thermal accelerometers designed for automated wirebonder assembly. 2008 IEEE 21st International Conference on Micro Electro Mechanical Systems, 2008: 880-883.

[114] J Qiu, J H Lang, A H Slocum. A centrally-clamped parallel-beam bistable MEMS mechanism. Technical Digest. MEMS 2001. 14th IEEE International Conference on Micro Electro Mechanical Systems (Cat. No. 01CH37090), 2001: 353-356

[115] J Li, G Ananthasuresh. A quality study on the excimer laser micromachining of electro-thermal-compliant micro devices. Journal of Micromechanics and Microengineering, 2001, 11(1): 38. 
[116] R J Chang, P W Shih, R Z Huang, et al. Application of piezo-driven polymer microgripper in automatic transportation of micro object. IEEE International Conference on Mechatronics, 2005. ICM'05., 2005: 140-144.

[117] Y Wu, G Ding, C Zhang, et al. Design and implementation of a bistable microcantilever actuator for magnetostatic latching relay. Microelectronics Journal, 2010, 41(6): 325-330.
[118] S Buetefisch, S Buettgenbach. New pneumatically actuated miniature gripper for micro assembly. Microrobotics and Microassembly III, 2001 : 32-39.

Submit your manuscript to a SpringerOpen ${ }^{\circ}$ journal and benefit from:

- Convenient online submission

- Rigorous peer review

- Open access: articles freely available online

- High visibility within the field

- Retaining the copyright to your article

Submit your next manuscript at $\boldsymbol{\nabla}$ springeropen.com 